\title{
Developmental and tumoral vascularization is regulated by G protein-coupled receptor kinase 2
}

\author{
Verónica Rivas, ${ }^{1,2}$ Rita Carmona, ${ }^{3}$ Ramón Muñoz-Chápuli, ${ }^{3}$ Marta Mendiola, 4 \\ Laura Nogués, 1,2 Clara Reglero,,1,2 María Miguel-Martín,4 Ramón García-Escudero,5 \\ Gerald W. Dorn II,6 David Hardisson,4,7 Federico Mayor Jr., 1,2 and Petronila Penela ${ }^{1,2}$ \\ 1Departamento de Biología Molecular and Centro de Biología Molecular "Severo Ochoa," Universidad Autónoma de Madrid, Madrid, Spain \\ 2 Instituto de Investigación Sanitaria La Princesa, Madrid, Spain. ${ }^{3}$ Department of Animal Biology, University of Málaga, Málaga, Spain.

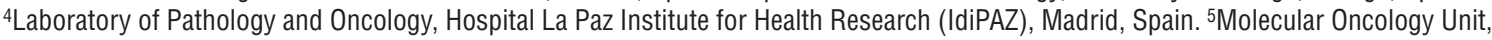 \\ Division of Biomedicine, Centro de Investigaciones Energéticas, Medioambientales y Tecnológicas, Madrid, Spain. \\ ${ }^{6}$ Washington University Center for Pharmacogenomics, St. Louis, Missouri, USA. ${ }^{7}$ Department of Pathology, Hospital Universitario La Paz, \\ School of Medicine, Universidad Autonoma de Madrid, IdiPaz, Madrid, Spain.
}

\begin{abstract}
Tumor vessel dysfunction is a pivotal event in cancer progression. Using an in vivo neovascularization model, we identified G protein-coupled receptor kinase 2 (GRK2) as a key angiogenesis regulator. An impaired angiogenic response involving immature vessels was observed in mice hemizygous for Grk2 or in animals with endothelium-specific Grk2 silencing. ECs isolated from these animals displayed intrinsic alterations in migration, TGF- $\beta$ signaling, and formation of tubular networks. Remarkably, an altered pattern of vessel growth and maturation was detected in postnatal retinas from endothelium-specific Grk2 knockout animals. Mouse embryos with systemic or endothelium-selective Grk2 ablation had marked vascular malformations involving impaired recruitment of mural cells. Moreover, decreased endothelial Grk2 dosage accelerated tumor growth in mice, along with reduced pericyte vessel coverage and enhanced macrophage infiltration, and this transformed environment promoted decreased GRK2 in ECs and human breast cancer vessels. Our study suggests that GRK2 downregulation is a relevant event in the tumoral angiogenic switch.
\end{abstract}

\section{Introduction}

The homeostasis and growth of vertebrate organisms requires a complex vascular network, necessary to deliver oxygen and nutrients to tissues and to allow waste disposal and immune cell surveillance (1). The process known as sprouting angiogenesis takes place during embryonic development to expand vasculogenic plexuses and postnatally for wound-healing repair and tissue remodeling. However, abnormal angiogenesis can also fuel inflammatory and tumoral diseases (2). The complex angiogenic response comprises finely orchestrated sequential steps (activation, resolution, and maturation) and is initiated and regulated by manifold factors from the VEGF, FGF, or PDGF families as well as by cytokines and chemokines $(1,3)$. Angiogenesis is triggered when quiescent ECs in preexisting vessels sense proangiogenic signals (such as VEGF, FGF, or sphingosine-1-phosphate [S1P]) and loose their intercellular junctions and when their basement membrane is degraded. Activated ECs guided by complex chemoattractant gradients of such proangiogenic factors proliferate and migrate out of the vessel wall. The resolution phase of sprouting includes cessation of EC migration and proliferation, restoration of cell-cell junctions for barrier function, PDGF-BB- and CXCL12-mediated recruitment of pericytes in order to stabilize the endothelial wall, and reconstitution of the basement membrane (1).

Chemokines and TGF- $\beta$ signaling play an important and multifaceted role in the timely coordination of activation and resolution phases required for the successful formation of functional, mature

Conflict of interest: The authors have declared that no conflict of interest exists. Citation for this article: JClin Invest. 2013;123(11):4714-4730. doi:10.1172/JCI67333. neovessels by recruiting stromal proangiogenic cells and also by directly modulating both endothelial and pericyte functions $(3,4)$. In particular, TGF- $\beta$ exerts positive and negative effects on the angiogenic process in a context-dependent way $(4,5)$. Such a proproliferative-to-antiproliferative/promigratory-to-antimigratory signaling switch of TGF- $\beta$ might relate to actions through 2 distinct receptors, ALK1 and ALK5, coexpressed in ECs in certain conditions (4). The precise role of TGF- $\beta$ in angiogenesis may depend on local cues, cell-cell interactions, and modulating factors impacting on ALK1/ALK5 interplay that need to be identified.

G protein-coupled receptor kinase 2 (GRK2) is a ubiquitous, essential protein kinase that is emerging as an integrative node in many signaling networks $(6,7)$. GRKs were initially identified as key players in the modulation of GPCRs. GRK-mediated phosphorylation of ligand-occupied receptors leads to binding of $\beta$-arrestins, which in turn promotes GPCR desensitization and internalization (8). Besides such a canonical role, GRK2 can also initiate alternative signaling pathways and participate in cellular processes related to cell cycle progression, survival, or cell migration by phosphorylating and interacting with non-GPCR partners (7).

Interestingly, the GRK2 "interactome" in different cell types includes several actors in vascular homeostasis and remodeling $(3,9)$. Besides regulating chemokine GPCRs, GRK2 phosphorylates PDGF receptors (10) and also modulates TGF- $\beta$ signaling in epithelial cells (11) or cardiac fibroblasts (12). GRK2 inhibits PDGF-dependent chemotactic signaling in VSMCs (13) and modulates both vasoconstrictory and vasodilatory responses of VSMCs $(14,15)$, whereas increased GRK2 attenuates NO production by sinusoidal ECs in the context of liver injury (16). However, the role of GRK2 

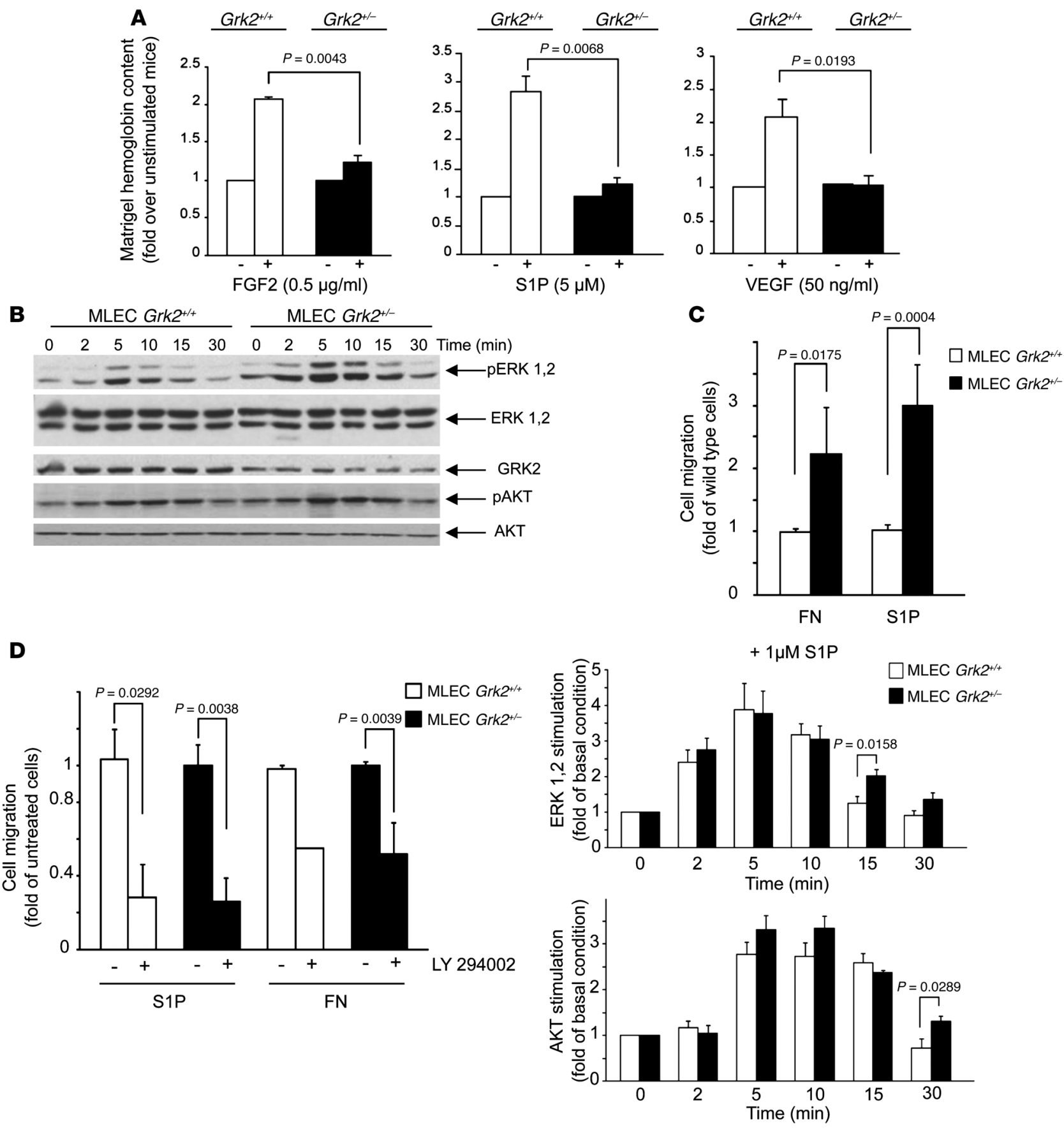

Figure 1

GRK2 deficiency results in an impaired in vivo angiogenesis, despite effective EC activation. (A) Matrigel implants mixed with the indicated stimuli or vehicle were injected into WT $\left(G r k 2^{+/+}\right)$or global hemizygous $\left(G r k 2^{+/-}\right)$mice, and hemoglobin content was quantified as described in the Methods. The angiogenic response data (calculated as fold over control conditions) were obtained from 9 to 12 animals for each condition in 3 to 4 independent experiments. (B) GRK2 downregulation does not compromise the S1P-induced activation of ERK1/2 and AKT pathways in MLECs. Data from 3 to 4 independent experiments are shown. Representative blots are shown. (C and D) GRK2 downmodulation enhances the PI3K-dependent directed cell migration of ECs in response to fibronectin (FN) and S1P. Data of 4 to 6 independent experiments performed in duplicate are shown. ERK $1 / 2$ and AKT activation and cell migration assays are detailed in Methods. 

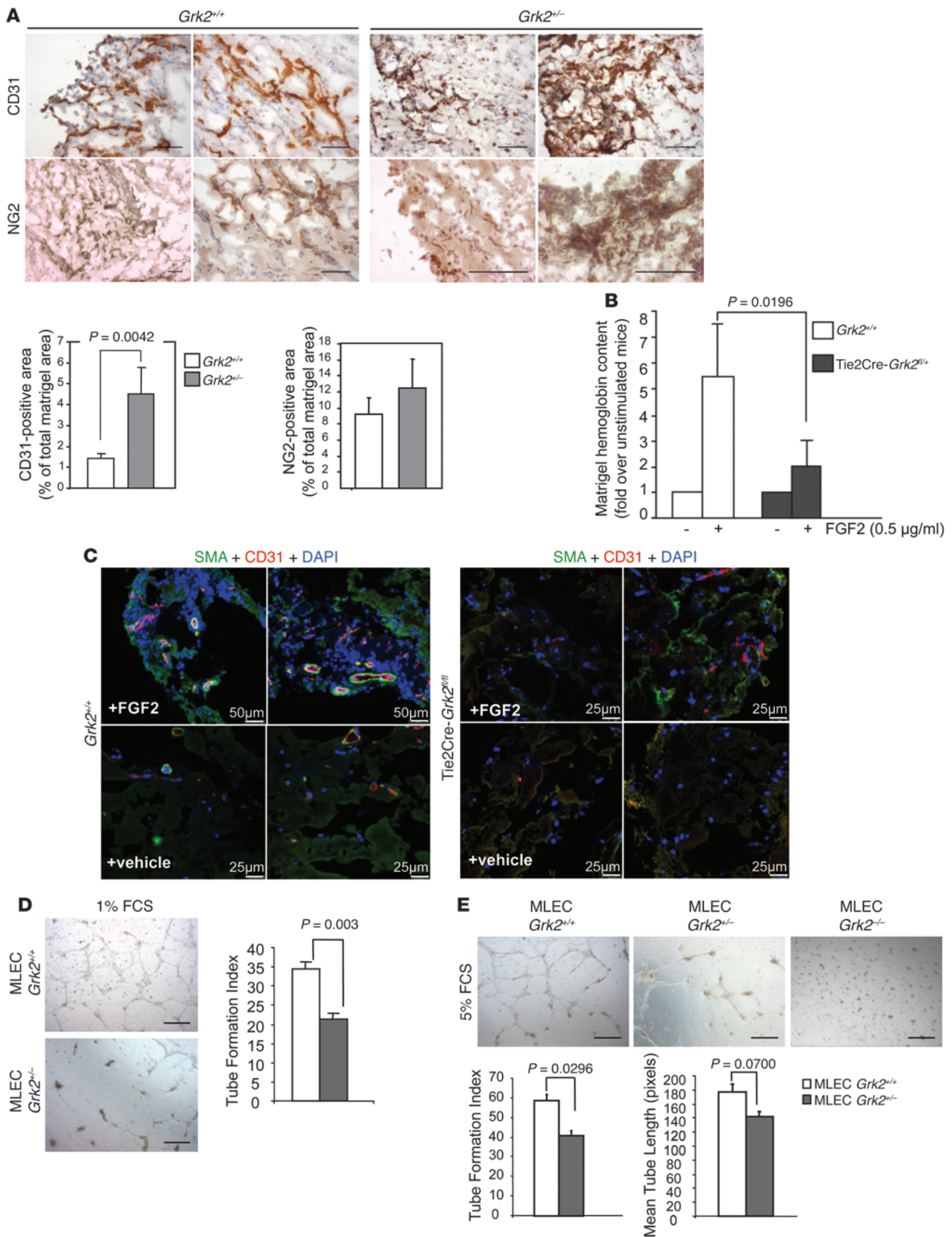


\section{Figure 2}

Endothelial GRK2 protein levels regulate vascular morphogenesis. (A-C) Angiogenesis in mice with global or endothelial-specific reduction of GRK2 expression is characterized by a lack of functional vessels, concomitant with the occurrence of aberrant vessel-like structures with a poor mural coverage. Sections of FGF2-embeded Matrigel plugs excised (A) from WT Grk2+/+ and global Grk2+/- or (C) from Tie2CreGrk2 $2^{f / f l}$ mice were analyzed by immunohistochemistry, and the density of invasion by either endothelial or mural cells was determined with (A) anti-CD31 or anti-NG2 or (C) SMA antibodies, respectively, as detailed in Methods. Ratios of CD31-positive cells to total DAPI-stained cells in FGF2-stimulated WT Grk2 $2^{+/+}$and Tie2Cre-Grk2 $2^{f / f l}$ mice were $0.22 \pm 0.06$ and $0.58 \pm 0.28$, respectively. (B) Endothelial GRK2 downmodulation is sufficient to impair the functionality of angiogenic vessels. The angiogenic response in WT or Tie2Cre-GRK2 $\mathrm{f} /+$ mice was determined as in Figure 1A. Data from 12 animals for each condition in 4 independent experiments are shown. (D and E) ECs with deficient GRK2 expression are less competent to form capillary-like networks in vitro. MLECs from WT $\left(\right.$ Grk2 $\left.2^{+/+}\right)$, global hemizygous $\left(\right.$Grk2 $\left.2^{+/}\right)$, or endothelial-specific (Tie2Cre-Grk2 ${ }^{f / f}$ ) GRK2 knockout mice were seeded on Matrigel-coated wells in the presence of (D) $1 \% \mathrm{FCS}$ or (E) $5 \%$ FCS. Photographs were taken after 16 to 18 hours in culture (original magnification, $\times 5$ ), and formation of tubular networks was quantified as described in Methods. Data were obtained from 2 to 3 independent assays performed in duplicate. Scale bar: $100 \mu \mathrm{m}$ (A); $25 \mu \mathrm{m}$ (C, Tie2Cre-Grk2 ${ }^{f / f l}$ and vehicle-treated Grk2 ${ }^{+/+}$); $50 \mu \mathrm{m}$ (C, FGF2-treated $G r k 2^{+/+}$); $500 \mu \mathrm{m}$ (D and E).

in vessel formation and stability in other pathophysiological settings has not been addressed.

We report herein that GRK2 is a relevant modulator of angiogenesis during development and tumor formation. GRK2 dosage is important in determining how ECs integrate different relevant physiological stimuli and to balance TGF- $\beta$ signaling to downstream pathways. Furthermore, ablation of GRK2 compromises postnatal angiogenesis and vascular remodeling in the retina and vasculogenesis during embryonic development as a result of defective vessel maturation, whereas GRK2 downregulation in ECs reduces pericyte coverage of tumoral vessels and potentiates tumor progression.

\section{Results}

GRK2 expression levels modulate the angiogenic response. To test whether GRK2 could impact the angiogenic process, we analyzed the in vivo neovascularization induced by Matrigel plugs mixed with the potent angiogenic inducers S1P, VEGF, or FGF2 subcutaneously injected in WT or hemizygous Grk2+/- mice (which express $40 \%-50 \%$ less kinase protein as compared with WT animals). As expected, each of these angiogenic factors induced hemoglobin accumulation in WT mice, as a readout of the occurrence of functional blood vessels. Interestingly, decreased expression of GRK2 resulted in marked reduction of hemoglobin content in response to either factor (Figure 1A), suggesting an alteration in vessel number and/or functionality.

We next analyzed whether such impaired angiogenic response could be caused by defective EC responsiveness using murine lung ECs (MLECs) isolated from WT or Grk2 $2^{+-}$mice. Neither AKT nor ERK activation was compromised in response to VEGF (Supplemental Figure 1A; supplemental material available online with this article; doi:10.1172/JCI67333DS1) or S1P (Figure 1B) in Grk2+/MLECs compared with that in WT cells but was instead modestly enhanced at longer times of stimulation (Figure 1B). Consistently, no significant changes in cell proliferation were noted between WT and $\mathrm{Grk2}^{+/-}$MLECs stimulated with angiogenic factors or with serum (Supplemental Figure 1B). As a major effect of S1P in vessel formation is to mobilize ECs, the influence of GRK2 levels on S1Pinduced chemotactic motility of MLECs was examined. Reduced GRK2 expression markedly enhanced migration toward S1P or fibronectin (Figure 1C), and a similar trend was also observed in response to VEGF (Supplemental Figure 1C). Both fibronectinand S1P-induced EC migration were abrogated by inhibition of the upstream AKT activator PI3K (Figure 1D), suggesting that modulation of AKT stimulation by GRK2 levels (see Figure 1B) might be crucial for motility in ECs.

Overall, these results suggested that changes in EC proliferation or migration were not related to the impaired angiogenic response observed in Grk2 $2^{+/-}$mice in the Matrigel plug assay. As functional vessel formation also requires endothelial tubular organization and concomitant pericyte coverage, vascular architecture was analyzed in sections of S1P- or FGF2-embedded Matrigel plugs. Capillarylike structures expressing both CD31 and SMA or NG2 as markers of endothelial and mural cells, respectively, were readily observed in WT animals (Figure 2A and Supplemental Figure 2A), consistent with the formation of mature vessels, but not in $\mathrm{Grk} 2^{+/-}$mice. In the later animals, CD31- or NG2-positive cells were distributed scatteredly or clustered in anarchic masses, suggestive of aberrant endothelial structures lacking mural coverage, despite the fact that endothelial and mural $\mathrm{Grk2}^{+/-}$cells infiltrate Matrigel plugs even better than those in WT animals (Figure 2A). This is consistent with the enhanced migration upon GRK2 downmodulation in MLECs (see above) or in isolated VSMCs toward the endothelialderived chemoattractant PDGF-BB (Supplemental Figure 1D).

Since no angiogenic phenotype has been described in mice with specific GRK2 depletion in VSMCs (15), we reasoned that aberrant angiogenesis in global Grk2 ${ }^{+/-}$mice might be caused by GRK2-triggered intrinsic defects of ECs. In support of this, endothelium-specific downregulation of GRK2 (in endotheliumspecific hemizygous mice) also resulted in an impaired angiogenic response in vivo (Figure $2 \mathrm{~B}$ ) and recapitulated vessel deficiency with an unstructured cellular network characterized by a sparse accumulation of endothelial and mural cells (Supplemental Figure 2B). An extra endothelium-specific decrease in GRK2 expression (Tie2Cre-Grk2 $2^{f l-}$ animals in Supplemental Figure 2, C and $\mathrm{D}$, or Tie2Cre-Grk2 $2^{f l f l}$ mice in Figure $2 \mathrm{C}$ ) further worsened this vascular phenotype in Matrigel implants.

These results indicated that altered GRK2 levels in ECs are sufficient to compromise key functions required for successful angiogenesis. Consistently, we found that the capacity of isolated MLECs to form "well-resolved" tubular networks in vitro was dampened by GRK2 downregulation in a dose-dependent manner (Figure 2, D and E). It is worth noting that a compensatory increase in the expression levels of other closely related GRK isoforms was not detected in MLECs from global GRK2 hemizygous mice (Supplemental Figure 2E). Tube length and tube formation index parameters (see Methods) were inherently impaired in Grk2 $2^{+-}$MLECs in low serum conditions (Figure 2D) and also in response to angiogenic stimuli (Figure 2E and Supplemental Figure 2F), pointing out that GRK2 levels can impact endothelial differentiation and remodeling.

TGF- $\beta$ signaling through ALK1 and ALK5 receptors in ECs is unbalanced upon GRK2 downregulation. Tubular morphogenesis requires coordination of manifold paracrine and autocrine signaling 

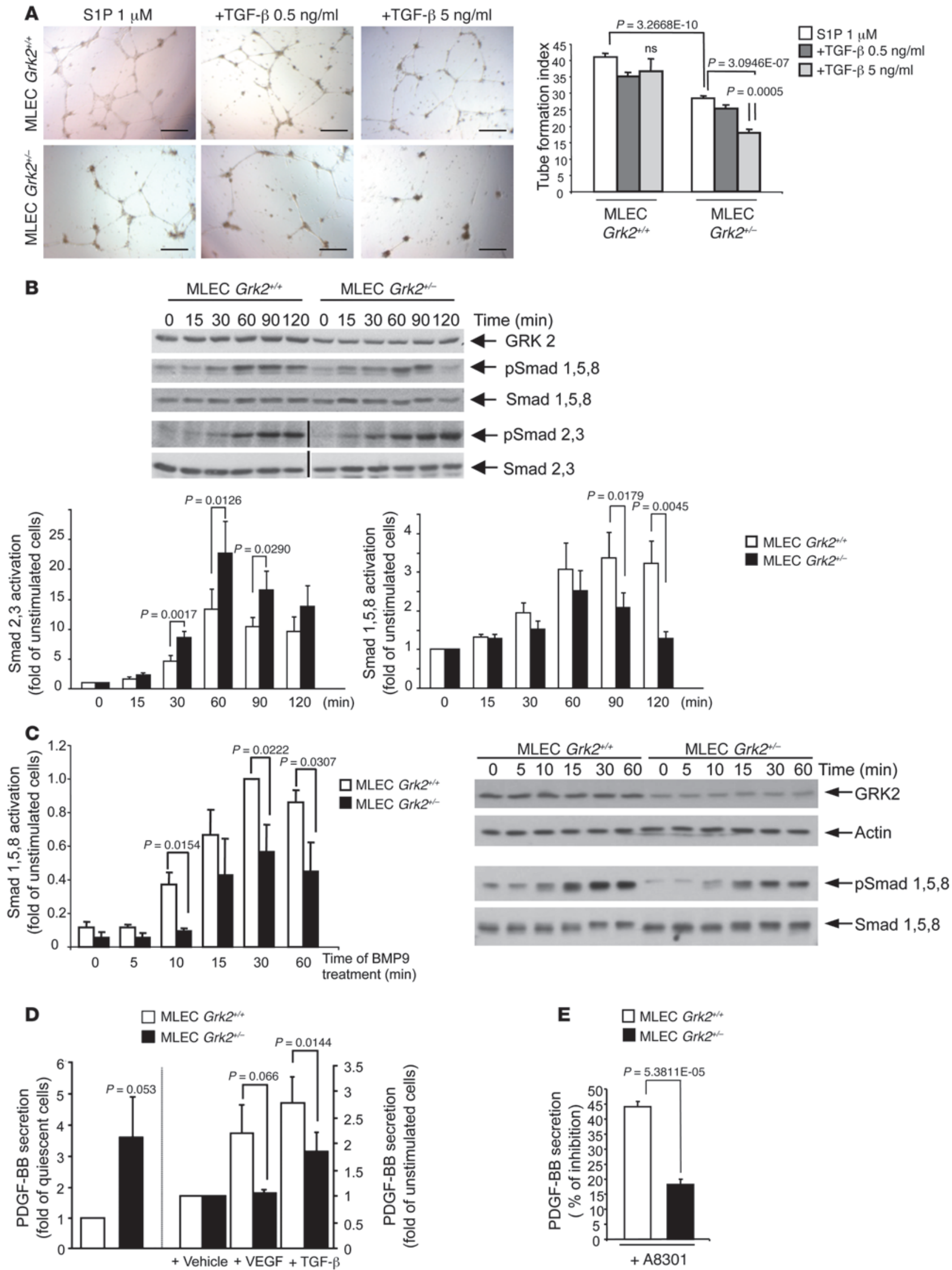

E $\square$ MLEC Grk2 $2^{\prime+}$

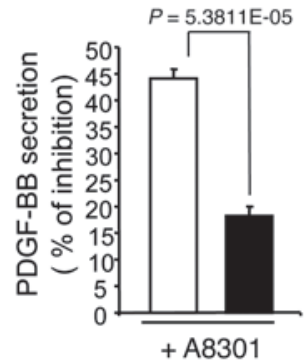




\section{Figure 3}

Effect of GRK2 downregulation on TGF- $\beta 1$ endothelial signaling. (A) ECs with reduced expression of GRK2 are more sensitive to dose-dependent inhibitory effects of TGF- $\beta 1$ on tube network formation. In vitro tube formation was monitored in the presence of $1 \mu \mathrm{M}$ S1P supplemented with or without 0.5 or $5 \mathrm{ng} / \mathrm{ml}$ TGF- $\beta 1$ and quantified as in Figure 2, D and E. Data from 3 independent assays performed in duplicate are shown. Scale bar: $500 \mu \mathrm{m}$. (B and C) Proper signaling of Smads downstream of ALK1 and ALK5 receptor activation depends on GRK2 expression. (B) Grk2+- MLECs display enhanced TGF- 31 -triggered activation of Smad2/3 paralleled by a decrease in Smad1/5/8 stimulation. The lanes were run on the same gel but, where indicated by the black vertical line, were noncontiguous. (C) BMP9-induced activation of Smad1/5/8 via ALK1 is reduced in Grk2+/- MLECs. (D) Both basal and angiogenic factor-induced PDGF-BB secretion are altered in Grk2+/- MLECs. Serum-starved cells were incubated for 48 hours in $1 \%$ FCS supplemented with or without $50 \mathrm{ng} / \mathrm{ml}$ VEGF or $5 \mathrm{ng} / \mathrm{ml}$ TGF- $\beta 1$, and PDGF-BB was determined in the cell-conditioned media as described in Methods. (E) The production of PDGF-BB in Grk2 ${ }^{+/-}$MLECs is less sensitive to ALK5 signaling inhibitors. Media of cells treated with $5 \mathrm{ng} / \mathrm{ml} \mathrm{TGF-} \beta 1$ for 48 hours in the presence or absence of the ALK5 inhibitor A8301 (5 $\mu \mathrm{M})$ was processed for PDGF-BB quantification. In B-E data from 3-4 independent experiments are shown.

factors. TGF- $\beta$ appears to be central to this process, as diverse angiogenic stimuli such angiopoietin 1 or PDGF control its production and secretion (17), and TGF- $\beta$ itself upregulates key angiogenic modulators such as S1P and FGF2 (18), VEGF (19), or PDGF-BB (20) in ECs, which altogether cooperate to build up vascular networks (5). Since reciprocal regulation of TGF- $\beta$ signaling and GRK2 expression has been reported in other cells types $(11,21)$, we analyzed whether GRK2 downregulation could alter TGF- $\beta$-dependent modulation of EC differentiation. Although contradictory TGF- $\beta$ activities on tube formation have been reported (5), in general, low doses of TGF- $\beta$ seem to promote a robust and better-organized capillary network, while high concentrations $(5-10 \mathrm{ng} / \mathrm{ml})$ restrict sprouting, proliferation, and tube-like aggregation $(18,22)$. In response to S1P, an abundant angiogenic component that accounts for the serum effects on EC proliferation/migration (23), the cord-like pattern of WT MLECs displayed similar characteristics and tube formation indexes (Figure $3 \mathrm{~A}$ ) as those promoted by complete serum (see Figure 2E), while the additional presence of increasing doses of TGF- $\beta$ did not impair formation of well-developed interconnecting tubes (Figure $3 \mathrm{~A}$ ). In contrast, tube formation index in Grk2 ${ }^{+/-}$MLECs was markedly reduced with the higher concentration of TGF- $\beta$ assessed, suggesting that a normal complement of GRK2 would be needed to counterbalance the inhibitory effects of TGF- $\beta$ signaling in angiogenesis. In keeping with this, Grk2+/MLECs were more sensitive than WT cells to the antiproliferative effects of high doses of TGF- $\beta$ (Supplemental Figure 3A).

It has been suggested that ALK1 activation by low concentrations of TGF- $\beta$ overcomes ALK5-mediated block of proliferation/migration (favoring the angiogenic activation phase). However, at high concentrations of TGF- $\beta$, ALK5 signaling predominates, thereby contributing to ulterior angiogenic resolution phases $(4,24)$. Interestingly, we found that specific TGF- $\beta$-dependent activation of ALK1 (monitored by phosphorylation of Smad1/5) was significantly attenuated in $\mathrm{Grk2} 2^{+/-}$MLECs compared with that in their WT counterparts (Figure 3B). In marked contrast, ALK5mediated phosphorylation of $\operatorname{Smad} 2 / 3$ was clearly upregulated in cells with lower GRK2 levels, suggesting that this protein might be instrumental in keeping the balanced TGF- $\beta$ signaling required for proper angiogenesis.

To gain further insight in the molecular mechanisms by which GRK2 levels modulate the highly interconnected ALK1/ALK5 pathways, we used specific modulators of such signaling routes. The enhanced stimulation of ALK5-dependent Smad2/3 phosphorylation observed in $\mathrm{Grk2}^{+/-}$MLECs was compatible with the previously reported direct inhibitory effect of GRK2 on Smad2/3 activity (11). In agreement with such a potential mechanism, Grk2 $2^{+-}$MLECs were less sensitive than their WT counterparts to inhibition of Smad2/3 activation by the specific Smad3 inhibitor SIS3 (Supplemental Figure 3B), whereas signaling in both cell types was similarly blocked by the upstream ALK5 inhibitor A8301 (Supplemental Figure 3B). Interestingly, in WT and Grk2 $2^{+/-}$MLECs, the presence of the SIS 3 inhibitor did not affect TGF- $\beta$-mediated stimulation of the ALK1-Smad1/5/8 branch (Supplemental Figure 3C). This result excludes a potential negative crosstalk from ALK5 to ALK1 at the Smad level and suggests the occurrence of additional mechanisms of GRK2 modulation. Consistent with this notion, phosphorylation of Smad1/5/8 in response to the specific ALK1 agonist BMP9 was also clearly hampered in Grk2 ${ }^{+/-}$MLECs compared with that in WT MLECs (Figure 3C). Overall, these data suggest that GRK2 levels would modulate TGF- $\beta$ signals in ECs by both inhibiting ALK5 signaling at the level of Smad2/3 and by positively contributing to the activation of the ALK1 route. The fact that we could not observe direct phosphorylation of ALK5 or ALK1 receptors by GRK2 (data not shown) further supports the notion that the targets of modulation lie downstream of the receptors.

GRK2 expression modulates the secretion profile of endothelial autocrine and paracrine factors. Tight control of PDGF-BB expression in ECs is important for proper mural cell coverage $(9,25)$ and this factor is upregulated by ALK5-Smad2/3 activation (20). Interestingly, basal secretion of PDGF-BB in quiescent ECs was circa 3- to 4-fold higher in $\mathrm{Grk2}^{+/-}$MLECs compared with that in WT cells (Figure 3D), whereas the former failed to further increase secretion of PDGF-BB in response to VEGF or TGF- $\beta$ as occurred in WT cells (Figure 3D). The basal secretion of PDGF-BB was markedly diminished in WT MLECs treated with the specific ALK5 inhibitor A8301 but not as efficiently in $\mathrm{Grk2}^{+/-}$MLECs (Figure 3E), consistent with ALK1/ ALK5 signaling imbalance upon GRK2 deficiency. In addition, the secretion pattern of antiangiogenic-to-proangiogenic-related factors was also unbalanced in quiescent Grk2 $2^{+/-}$MLECs, with diminished levels of angiostatic factors and molecules involved in the maturation phases of angiogenesis, such as IL-13, PF4, IP-10, or TSP1 (Supplemental Figure 3D). Consistent with the fact that PDGF-BB expression during angiogenesis seems to be restricted to immature endothelium (26), Grk2+- MLECs formed stable, mature vascular structures less proficiently, as also suggested by the increased permeability of their endothelial monolayers compared with those of WT cells (Supplemental Figure 3E). Thus, improper/untimely endothelial secretion of PDGF upon GRK2 downregulation might determine the defective attachment of mural cells to endothelial angiogenic structures observed in systemic or endothelium-specific GRK2 hemizygous mice.

Endothelial downregulation of GRK2 levels leads to an altered pattern of vessel growth and maturation in the postnatal mouse retina. In order to shed light on the functional impact of altered GRK2 levels in ECs on the angiogenic process, we used the mouse retina model, which has been extensively used to investigate both physi- 
A
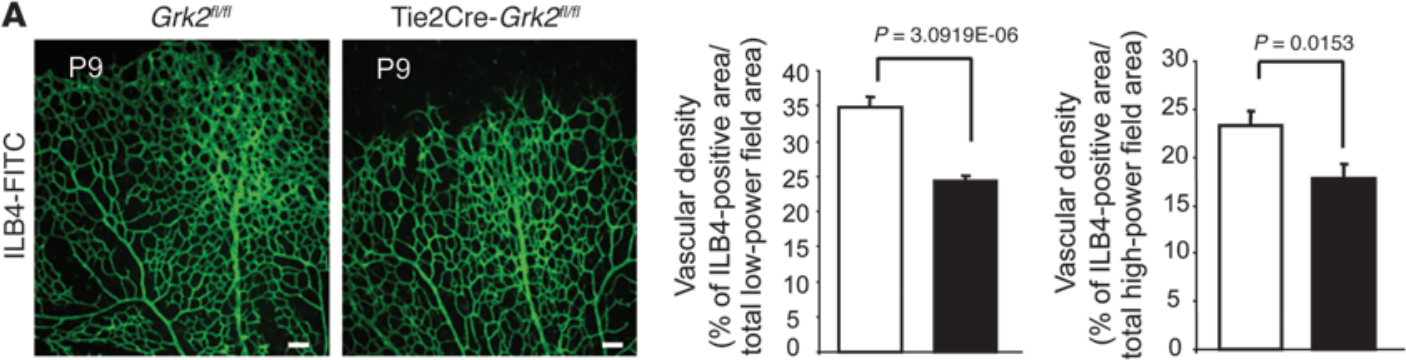

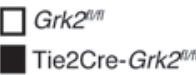

Retinal vessel outgrowth

B

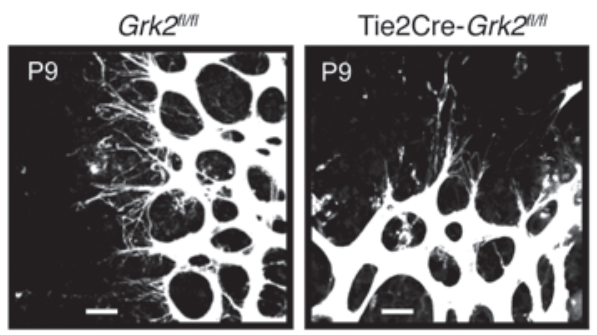

C
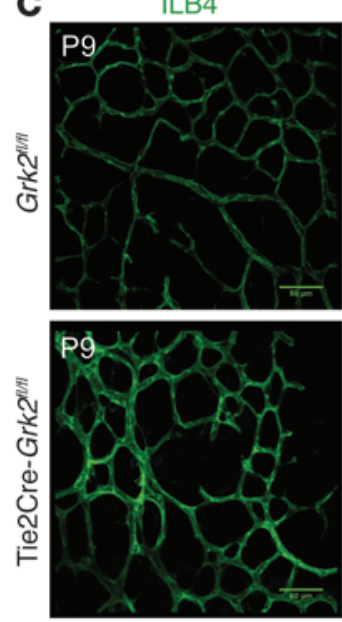

D
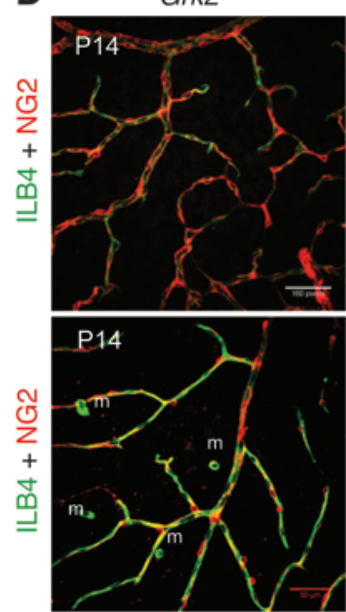

NG2
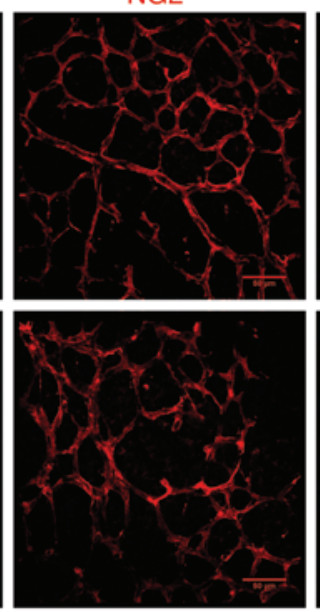

Tie2Cre-Grk2
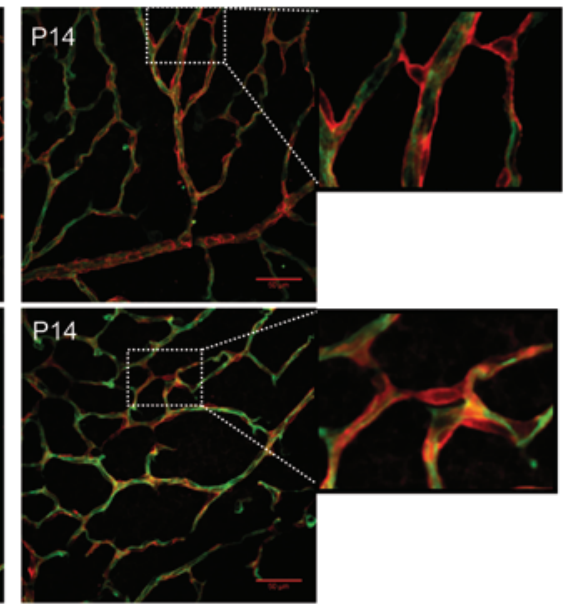

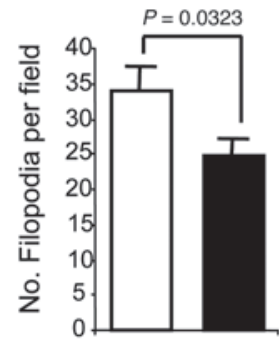

ILB4 + NG2

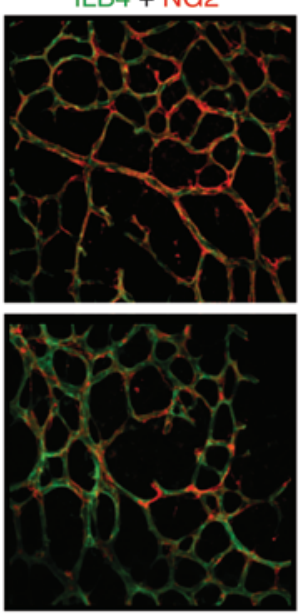

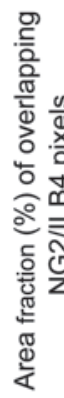

$\square$ Grk2"n

Tie2Cre-Grk2 ${ }^{\text {mn }}$

\section{Figure 4}

Loss of endothelial GRK2 impairs developmental outgrowth and maturation of the retinal vasculature. (A and $\mathbf{B}$ ) Expansion of the primary vascular plexus and endothelial filopodia was reduced in the absence of GRK2. (A) Whole-mount P9 retinas from WT (Grk2 $\left.{ }^{f l / f i} ; n=5\right)$ or Tie2Cre-Grk2 ${ }^{f l / f l}$ $(n=5)$ pups were stained with endothelial FITC-ILB4 marker. The ILB4-positive area was quantified in the entire retinal surface (low magnification) or in proximal regions of the vascular plexus (high magnification) (WT, $n=15$; mutant, $n=12$ ) as detailed in Methods. (B) Filopodia of tip cells were counted in high-power fields of 5 WT retinas $(n=17)$ and 6 Tie2Cre-Grk ${ }^{f / f l}$ retinas $(n=18)$. (C and D) Delayed recruitment of pericytes to retinal ECs and perturbed cell-cell interactions in mutant mice. Whole-mount (C) P9 and (D) P14 retinas of WT $(n=5)$ and Tie2Cre$\mathrm{Grk}^{f / f l}(n=5)$ mice were double stained with ILB4-FITC and anti-NG2 antibodies, and the corresponding positive areas were quantified ( $n=14$ and $n=11$ images, respectively) as detailed in Methods. (C) Pericyte coverage was expressed as the percentage fraction of colocalizing pericyte- and endothelial-positive areas. (D) Abnormal association of pericytes with endothelial capillaries in the primary vascular plexus of P14 Tie2Cre-Grk2 ${ }^{f / f t}$ retinas. Some pericytes remain dissociated from ECs and make irregular connections between endothelial capillaries (zoomed images). High-magnification fields ( $n=12$ from retinas of $5 \mathrm{Grk} 2^{\mathrm{fl} / \mathrm{fl} \text {; }}$ $n=20$ from retinas of 5 Tie2Cre-Grk2 $\left.{ }^{f / f f}\right)$ were inspected. $m$, macrophages. Scale bar: $100 \mu \mathrm{m}$ (A, C, and D); $25 \mu \mathrm{m}$ (B). 


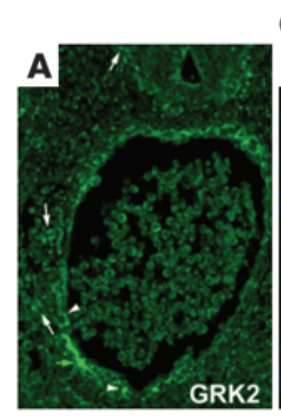

GRK2 expression in vascular development
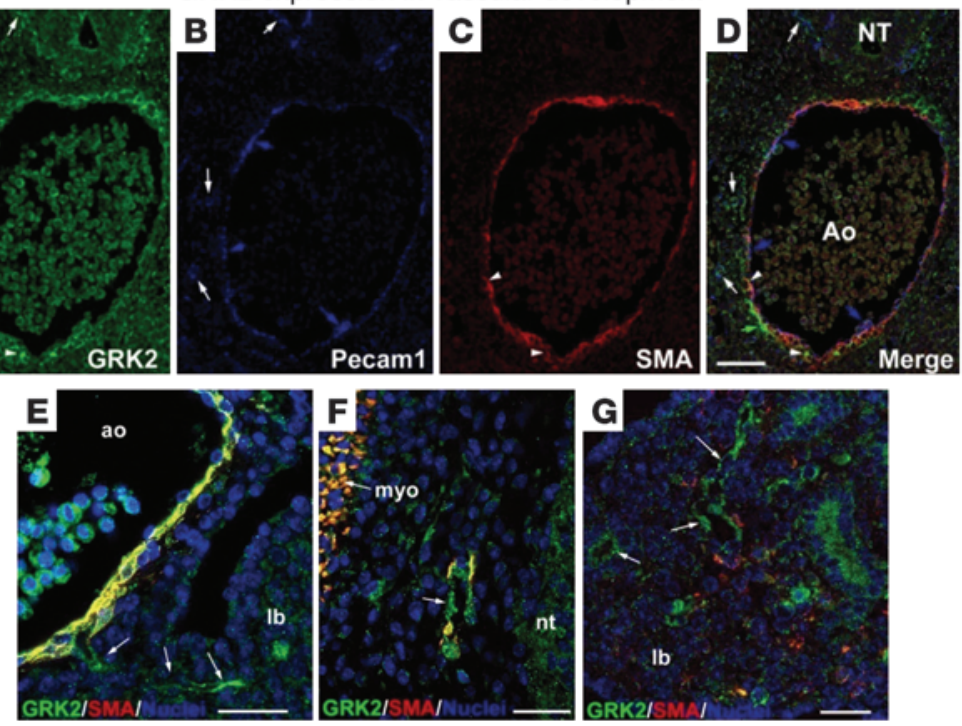

Vascular development in Grk2- embryos
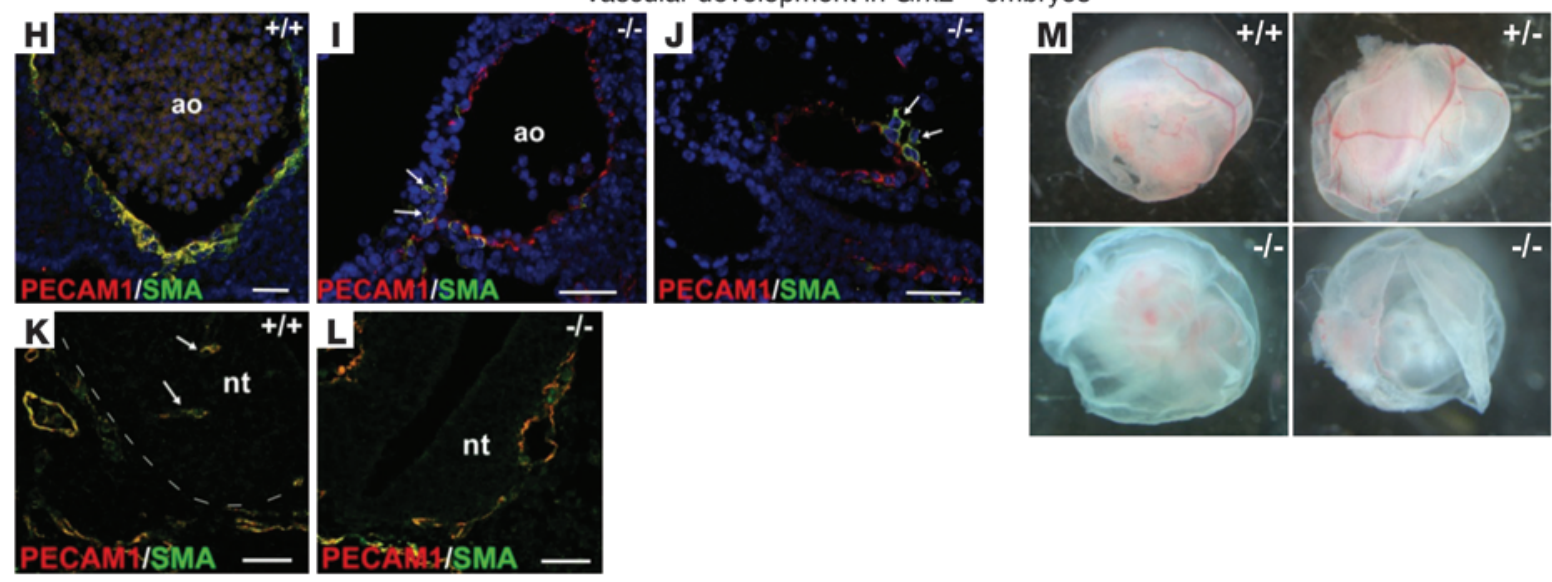

Figure 5

Abnormal vascular network formation and morphology in global GRK2 knockout embryos. (A-D) Vascular expression pattern of GRK2 in WT embryos. Triple immunostaining of (A) GRK2 and (B) endothelial and (C) smooth muscle markers in an E10.5 Grk2+/+ embryo. GRK2 is expressed in cells around the aorta (ao), colocalizing with SMA (arrowheads). Most aortic endothelium is GRK2 negative (blue arrows), while GRK2 colocalizes with PECAM1 in some small vessels (white arrows). (E) A small GRK2-positive vessel emerging from the aorta toward the lung bud (Ib) in an E11.5 embryo (arrows). By this stage, colocalization of GRK2 with SMA is more prominent in the aortic media. (F and G) GRK2-positive ECs in small vessels of the perineural plexus around the (F) neural tube (nt) and (G) lung bud from an E11.5 embryo. Note GRK2/SMA colocalization in the myotome (myo). (H-J) Vascular phenotype of E10.5 GRK2-null (Grk2 $2^{--}$) embryos. (H) In contrast to normal aortic wall architecture in the WT embryo, (I and $\mathbf{J}$ ) the wall of large- and medium-sized vessels of the GRK2-null embryos was constituted of only PECAM-positive ECs, with few SMA-positive cells loosely connected to the endothelium (arrows). (K and $\mathbf{L}$ ) Lack of angiogenic growth of vessels (arrows) within the neural tube of (L) GRK2-null compared with (K) WT embryos. Note the disorganized vessel wall of the perineural plexus in the mutant embryo. (M) Wholemount view of E10.5 yolk sacs isolated from Grk2+/+, Grk2+/-, and $\mathrm{Grk2}^{-/-}$embryos. Large blood vessels are completely absent in Grk2 $2^{-/-}$mice. Scale bar: $25 \mu \mathrm{m}(\mathbf{A}-\mathbf{J}) ; 50 \mu \mathrm{m}(\mathbf{K}$ and $\mathbf{L})$.

ologic and pathologic angiogenesis (27). We observed defective vessel outgrowth in the P9 retinas from Tie2Cre-Grk $2^{f l / f l}$ mice, characterized by reduced vascular density and delayed radial expansion of the superficial vascular plexus (Figure 4A). A similar trend was noted upon endothelial marker quantification in high-power fields of central areas of the capillary plexus (Figure 4A). Moreover, the number of loops in the retinal vasculature of Tie2Cre-Grk $2^{f l / f l}$ mice was clearly reduced compared with that of WT animals (Supplemental Figure 4A), pointing to a deficient branching activity and consistent with a reduced vascular density in mutant P9 retinas. Consistently, sprouting activity (measured by the number of filopodia per field) was significantly impaired at the vascular front in $\mathrm{P} 9$ retinas depleted of endothelial GRK2 (Figure 4B). In addition, both the lower presence of NG2-positive cells in the retinas of P9 Tie2Cre-Grk2fl/fl pups (Supplemental Figure 4B) and the reduced extent of endothelial/pericyte surface colocalization compared with that in WT animals (Figure 4C) suggested marked defects in pericyte recruitment to retinal vessels and inadequate endothelial coverage upon GRK2 downregulation. 
Interestingly, in the P14 retinas, radial expansion of capillary plexus and pericyte/endothelial ratios appeared to be similar in Tie2CreGrk2fl/fl retinas compared with that in WT animals (Supplemental Figure 4, C and D), suggesting that defective vascular growth was solved over time in the mutant animals. However, a careful analysis revealed that the full maturation of retinal vasculature was still perturbed in the absence of endothelial GRK2 expression, as vascular remodeling was deficient and/or delayed. In the mouse retina, vasculature is remodeled from an initially exuberant interconnected vessel meshwork into a simplified architecture that facilitates efficient blood flow (28). Regression of capillaries results in the formation of capillary-free zones around arteries with a marked reduction in vascular density. Notably, such reduction was not achieved in Tie2Cre-Grk $2^{f l / f l}$ retinas at $\mathrm{P} 14$, when pruning of the primary plexus was almost completed in control animals (ref. 29 and Supplemental Figure 4C). Moreover, vascular loops were irregularly formed in Tie2Cre-Grk $2^{l / f l}$ mice compared with those in WT mice, as shown by the higher branching detected in P14 Tie2Cre-Grk2flfll retinas (Supplemental Figure 4C). All these alterations were suggestive of an impaired functional interaction between ECs and pericytes. In keeping with this, $\mathrm{P} 14$ retinas of Tie2Cre-Grk $2^{f / f l}$ mice displayed pericytes that were abnormally associated with endothelial vessels, connecting adjacent vessels without an underlying endothelial track (Figure 4D).

Overall, our data suggested that endothelial GRK2 was a key modulator of vessel outgrowth in the mouse retina and that its downmodulation impaired the establishment of proper functional EC-pericyte interactions, resulting in impaired timing of vascular remodeling and selective vessel regression.

GRK2 deficiency leads to abnormal vessel formation in the mouse embryo, with decreased recruitment of vascular pericytes. To further substantiate that GRK2 expression was critical for vascular functionality in physiological contexts, we analyzed the development of embryonic vasculature in the absence of GRK2. We first investigated the expression pattern of GRK2 in vessels of E9.5-E11.5 WT mouse embryos using different anti-GRK2 antibodies. Perivascular cells were consistently positive for GRK2 staining, and the GRK2 label colocalized with smooth muscle cell markers in large vessels such as the aorta (Figure 5, A and D, and Supplemental Figure 6A) more prominently by E11.5 (Figure 5E). GRK2 expression in the endothelium was more dynamical, with widespread staining in small-diameter vessels, in vessels emerging from the aorta, or in the perineural plexus (Figure 5, A-G, white arrows), whereas large vessels displayed patchy immunoreactivity, with a number of ECs being negative for GRK2 expression (Figure 5D, blue arrows, and Supplemental Figure 6A). This suggests that GRK2 expression would be upregulated in the endothelium undergoing normal physiological angiogenesis.

Interestingly, vasculature was markedly altered in systemic Grk2-/mouse embryos compared with that in WT embryos. At E9.5 and E10.5, gross malformations were observed, along with clear defects in perivascular cell recruitment, which gave rise to large vessels such as the aorta being lined essentially only by ECs. Perivascular SMA-positive immunoreactive cells were scarce and usually showed little contact with ECs (Figure 5, H-J, and Supplemental Figure 5, E-H). Purely angiogenic vessel growth was also impaired, as shown by the lack of vascular invasion of the neuroectoderm in the knockout embryos (Figure 5, K and L, and Supplemental Figure 5, A-D). In the Grk2 $2^{--}$embryos, vessels of the perineural plexus were markedly dilated around the neural tube (Figure 5L) and around the encephalon (Supplemental Figure 5, C and D).
Other vessels in Grk2-/- animals adopted irregular shapes, leading to marked asymmetry in the paired aorta (Supplemental Figure 5, G-J) and to abnormal vascularization in the limbs (Supplemental Figure 5, K and L). Some large vessels even displayed discontinuities in the endothelial layer, which could explain some occasional hemorrhages found in GRK2-deficient embryos (Supplemental Figure 5J and Supplemental Figure 6B). Moreover, expansion of the primitive capillary plexus of yolk sacs was severely compromised in Grk2-/- embryos (Figure 5M and Supplemental Figure 6C). In parallel to such generalized defects in both capillaries and large vessels, Grk2 $2^{-/}$embryos showed noticeable mesenchymal apoptosis in different regions at E10.5 (Supplemental Figure 6D).

Remarkably, embryos with selective genetic ablation of GRK2 in the endothelium (Tie2CRE-Grk2flfl embryos) also displayed vascular abnormalities at E10.5 to E11.5, consisting of poor and sparse mural coverage of large vessels. Contrary to that in WT mice, endothelia of the aortas did not show appropriate covering by WT SMA-positive cells (Figure 6, A and B). Some degree of interindividual variability in the extent of mural coverage was observed in Tie2-targeted GRK2 knockout embryos, which might reflect an incomplete Cre-dependent inactivation of floxed GRK2 alleles, as inferred by a patchy staining of endothelial GRK2 in knockout embryos (Figure 6, C and D). Similar to systemic Grk2-/animals, we observed that endothelial-restricted GRK2 knockout mice displayed scarce vascularization of the neuroectoderm compared with that in WT littermates (Figure 6, E and F), dilated vessels with irregular shapes in hindbrain and limbs (Figure 6, E-H), and asymmetry in the paired aortas (Figure 6I). All these findings were consistent with altered vessel maturation and impaired recruitment of mural cells taking place in this experimental model. Interestingly, despite their blood vessels seeming to be similarly malformed and immature, Tie2Cre-Grk2fl/fl embryos did not show apoptosis to the extent observed in global GRK2 knockout embryos, suggesting that extravascular defects would contribute to such process in the latter animals. Consistently, vascular anomalies in endothelium-specific GRK2 knockout embryos did not impede their development and postnatal survival. However, Tie2Cre-Grk $2^{f / f l}$ mice showed vascular malformations at adulthood (Figure 6, J-S). In some animals the microvasculature of the heart displayed probable microhemorrhages, as suggested by the intratissular blood accumulation not surrounded by endothelium (Figure 6Q). This is indicated by the lack of PECAM and laminin staining (Figure 6, Q and R). Blood vessels in liver were also affected, showing increased capillary size, reduced mural coverage, and disorganized vascular pattern (Figure 6, $\mathrm{O}$ and $\mathrm{P}$ ). The above results clearly demonstrated that endothelial GRK2 is pivotal for the control of vascular maturation by influencing the extent of mural cell recruitment and endothelial coverage.

Impaired mural cell recruitment and increased size of tumor vessels correlates with tumor growth upon GRK2 downregulation in vivo. Tumor microvasculature is usually highly angiogenic and leaky, with enlarged and dilated vessels of irregular contours lined by immature walls as a result of pericyte loss and diminished deposition of basement membrane (30). Since many of the hallmarks of tumor vessels were recapitulated upon ablation or downregulation of endothelial GRK2 levels, we investigated whether the pattern of vessel formation in tumors and tumor growth was influenced by the extent of GRK2 expression in ECs. B16F10 melanoma cells were implanted subcutaneously in global hemizygous GRK2 mice with different levels of endothelial GRK2 (Grk2 $2^{+/-}$mice retaining $50 \%$ of 

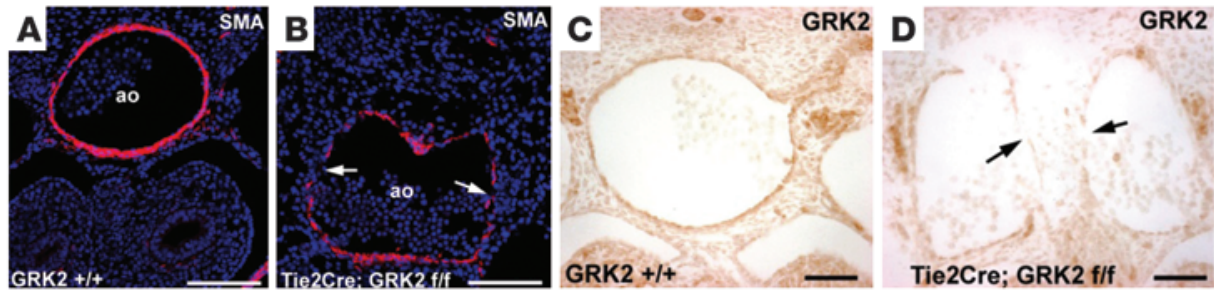
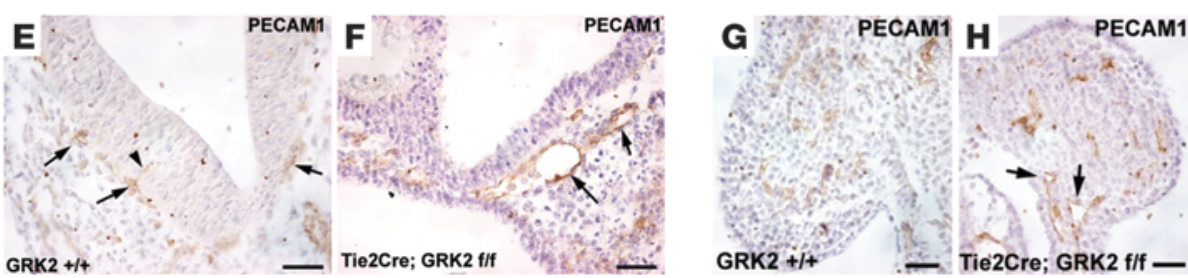

SMA
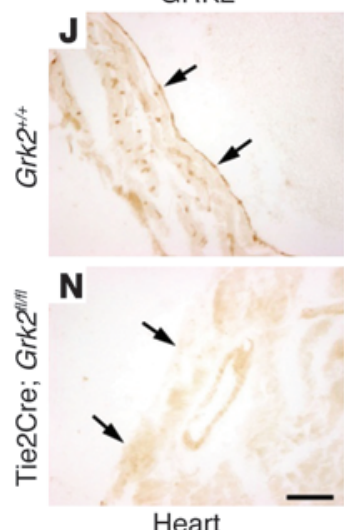

Heart
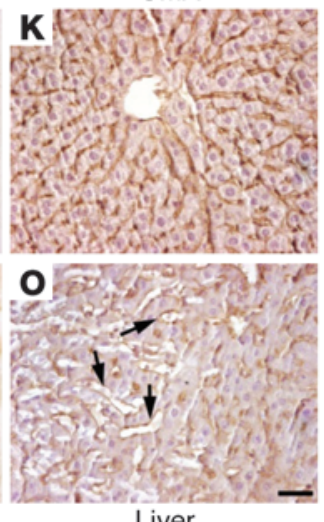
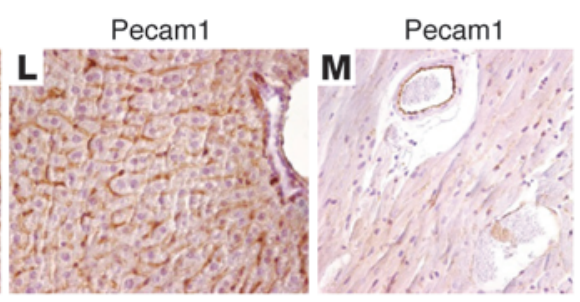

Liver

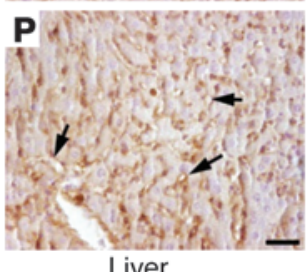

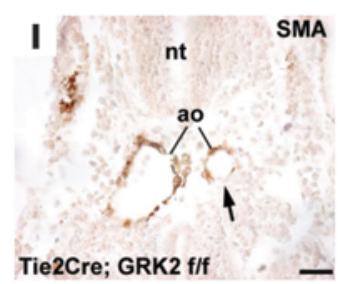
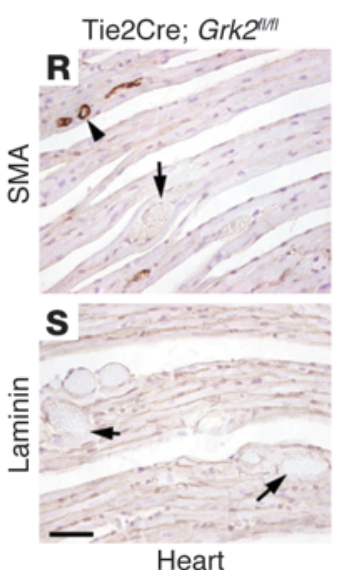

Figure 6

Specific downregulation of endothelial GRK2 expression causes vascular malformations during embryonic development and at adulthood. (A-D) Endothelial-specific ablation of GRK2 leads to a delayed formation of the aortic media. GRK2 immunoreactivity is reduced in endothelium of E11.5 embryos (arrows in D), while GRK2 staining of nervous ganglia adjacent to the aorta is unaffected. The aortic endothelium shows defective covering of SMA-positive cells (arrows in B) in mutant animals. (E-I) Vascular defects in E10.5 embryos upon specific ablation of endothelial GRK2. Angiogenesis of the hindbrain is delayed in Tie2Cre-Grk2 ${ }^{f / f l}$ embryos. PECAM1-positive vessels can be seen within the neuroectoderm of control embryos (arrowhead in E), but they are scarce or absent in Tie2Cre-Grk2 $2^{f / f l}$ embryos (arrows in E-I indicate representative vessels). Vessels of the perineural plexus (arrows in $\mathbf{E}$ ) and of the limb bud (arrows in $\mathbf{H}$ ) are dilated in the mutant embryo as compared with the WT (F and $\mathbf{G}$, respectively). The paired aorta from this Tie2Cre-Grk2 fl/fl embryo also shows a reduced left branch (arrow in I), similar to that of the systemic mutant (see Supplemental Figure 5). (J-S) Adult mice with endothelial-specific ablation of GRK2 show disorganization and dilatation of small vessels and capillaries in the liver (arrows in $\mathbf{O}$ and $\mathbf{P}$ ). GRK2 staining is strongly reduced in the cardiac endocardium (compare to arrows in $\mathbf{J}$ and $\mathbf{N}$ ) and coronary endothelium of Tie2Cre-Grk2 ${ }^{f / l f l}$ mice. Extravascular blood accumulations without PECAM1-positive endothelium covering (arrows in $\mathbf{Q}$ ) and lacking SMA and laminin immunoreactivity (arrows in $\mathbf{R}$ and $\mathbf{S}$ ) suggests microhemorrhages. A normal coronary vessel is shown (arrowhead in R). Scale bar: $25 \mu \mathrm{m}(\mathbf{C}-\mathbf{F}) ; 50 \mu \mathrm{m}(\mathbf{A}, \mathbf{B}, \mathbf{G}-\mathbf{I}, \mathbf{K}, \mathbf{L}, \mathbf{O}$, and P); $100 \mu \mathrm{m}(\mathbf{J}, \mathbf{M}, \mathbf{N}$, and Q-S).

GRK2 protein at endothelium or Tie2Cre-Grk2 ${ }^{f /-}$ mice, expressing approximately $25 \%$ of this protein; Supplemental Figure 2D), and subsequent evolution of tumors was compared with that in WT animals. The "quality" of the tumor vessels driven by B16F10 melanoma cells was particularly poor in Grk2 $2^{+/-}$mice, causing irregular blood perfusion (Supplemental Figure 7A). We observed that tumors grown in mice with lower endothelial levels of GRK2 consistently reached larger sizes in a dose-dependent manner (Figure 7A). Staining of tumor sections with EC markers demonstrated an increase in the proportion of large and dilated vessels (Figure 7, $\mathrm{B}$ and $\mathrm{C}$ ) in tumors grown in mice displaying reduced GRK2 protein at endothelium. Moreover, tumors implanted into Tie2Cre-Grk $2^{f /-}$ mice contained vessels with a substantial reduction in colocalization of SMA-positive cells (Figure 7D). Quantification of mural cell coverage revealed circa $90 \%$ reduction in these animals compared with that in WT animals (Figure 7E). We investigated whether such deficient pericyte-endothelial attachment would favor extravasation of immune cells such as macrophages, which positively contribute to tumor growth (31). Interestingly, we observed a higher infiltration of macrophages in tumors grown in Tie2Cre-Grk $2^{f /-}$ mice (Figure 7F). Thus, we investigated in more detail this process and its potential contribution to enhanced tumor growth in endothelium-deficient GRK2 mice. The conditioned medium of MLECs isolated from Tie2Cre-Grk2 $2^{f / f l}$ animals displayed an enhanced ability to promote macrophage migration compared with that of WT MLECs (Figure 8A), likely related to the increased basal secretion of CXCL12 (Supplemental Figure 7B) and of other macrophage regulatory and chemoattractant factors, such as GM-CSF, KC, or 
A

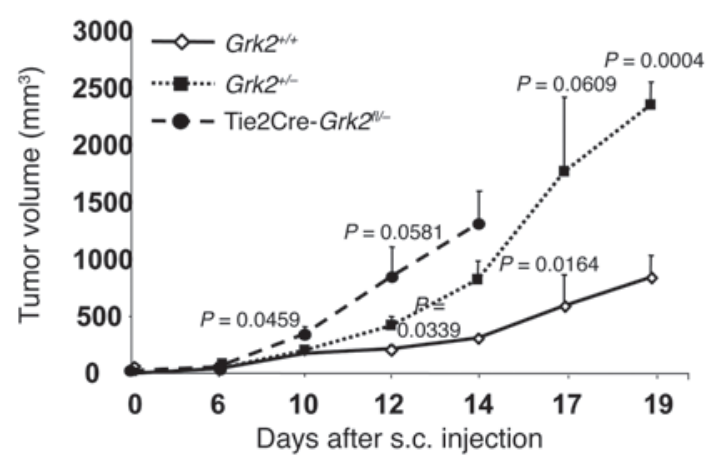

C

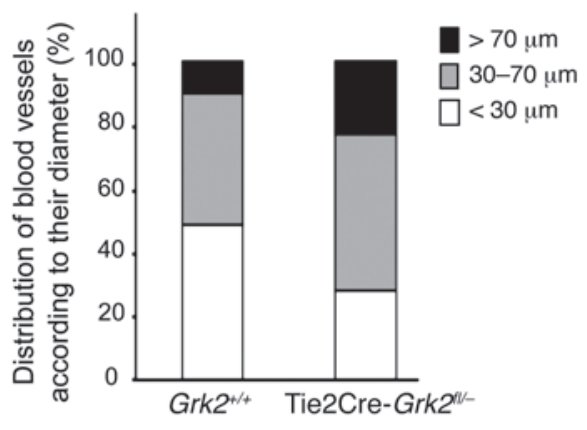

B

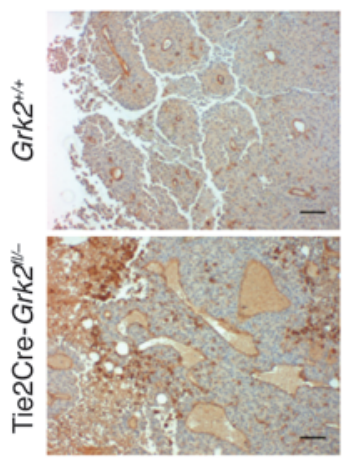

ILB4 staining

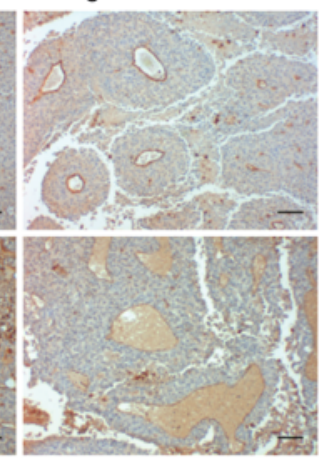

D

Grk2 $^{+1+}$

Tie2Cre-Grk2 $2^{n-1}$
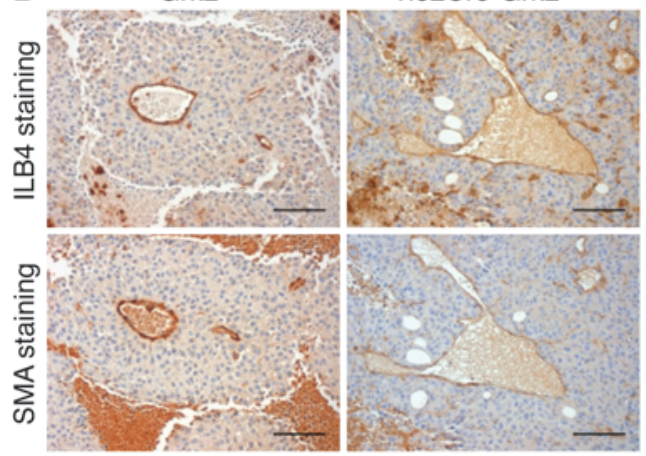

E

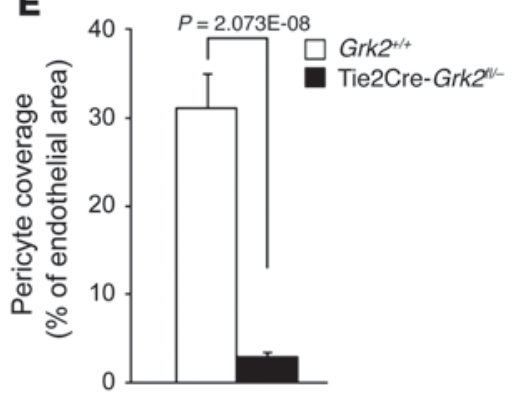

F

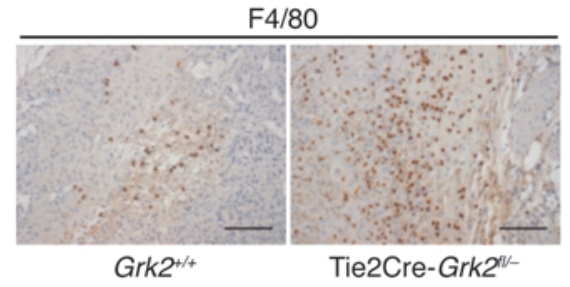

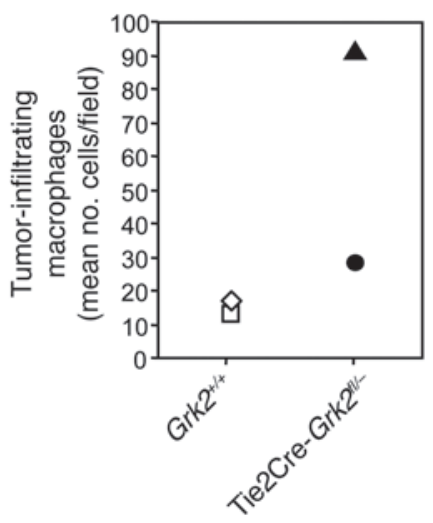

\section{Figure 7}

Endothelial GRK2 levels influence tumor growth by altering pericyte recruitment and tumor microvasculature. (A) Tumors implanted in mice with reduced endothelial expression of GRK2 exhibit higher growth rates. B16F10 melanoma cells were subcutaneously injected in mice and tumor size was monitored as described in Methods ( $n=3-4$ animals for each condition in 3 independent experiments). $P$ values indicated in comparisons between WT and hemizygous mice and between hemizygous and Tie2Cre-Grk2 ${ }^{\mathrm{ft} /}$ mice. (B and $\left.\mathbf{C}\right)$ Higher occurrence of large and dilated vessels in tumors grown in Tie2Cre-Grk2 ${ }^{\text {tll- }}$ mice. Tumor sections were stained with the endothelial biotinylated ILB4 marker and hematoxylin counterstained. Vessel size was calculated as detailed in Methods. Distribution of vessel diameter in each condition is shown in C. (D and E) Reduced pericyte recruitment in tumors implanted in Tie2Cre-Grk2 $2^{f / l}-$ mice. Sections of tumors were (D) stained with biotinylated ILB4 and antiSMA antibodies or (E) double stained with fluorescein-ILB4 and anti-SMA antibodies to quantify the mural coverage as described in Methods. Data were expressed as a percentage of the total ILB4-positive area. (F) Tumors grown in Tie2Cre-Grk2 ${ }^{\text {fll- }}$ mice show increased macrophage density compared to those implanted in WT mice. The number of F4/80-positive cells in different sections covering the whole tumor mass is shown. Two distinct specimens were analyzed for each condition. Scale bar: $100 \mu \mathrm{m}$.

Factor-III (Supplemental Figure 7C), detected in ECs upon GRK2 downregulation. On the other hand, in line with data shown in Figure 7F, B16F10-derived tumors grown in Tie2Cre-Grk $2^{f / f l}$ mice showed a marked increase of intratumoral hypoxia (indicative of altered vascularization) and of the tumor-associated macrophagederived factor adrenomedullin (Figure 8B). Moreover, consistent with an important role for enhanced macrophage infiltration in this experimental model, tumor growth was favored by the presence of extra injected M2-polarized RAW267.4 macrophages in both WT mice and mice deficient of GRK2 in endothelia (Figure 8, C and D), and depletion of endogenous macrophages upon clodronate treatment abrogated the marked differences in tumor growth observed 
A

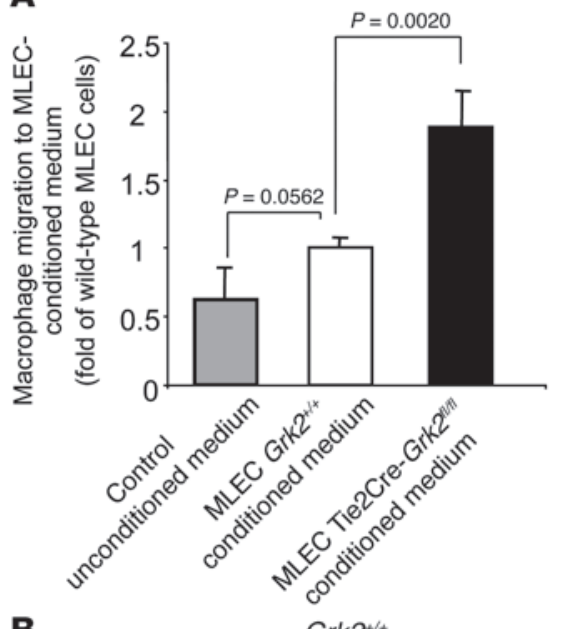

B
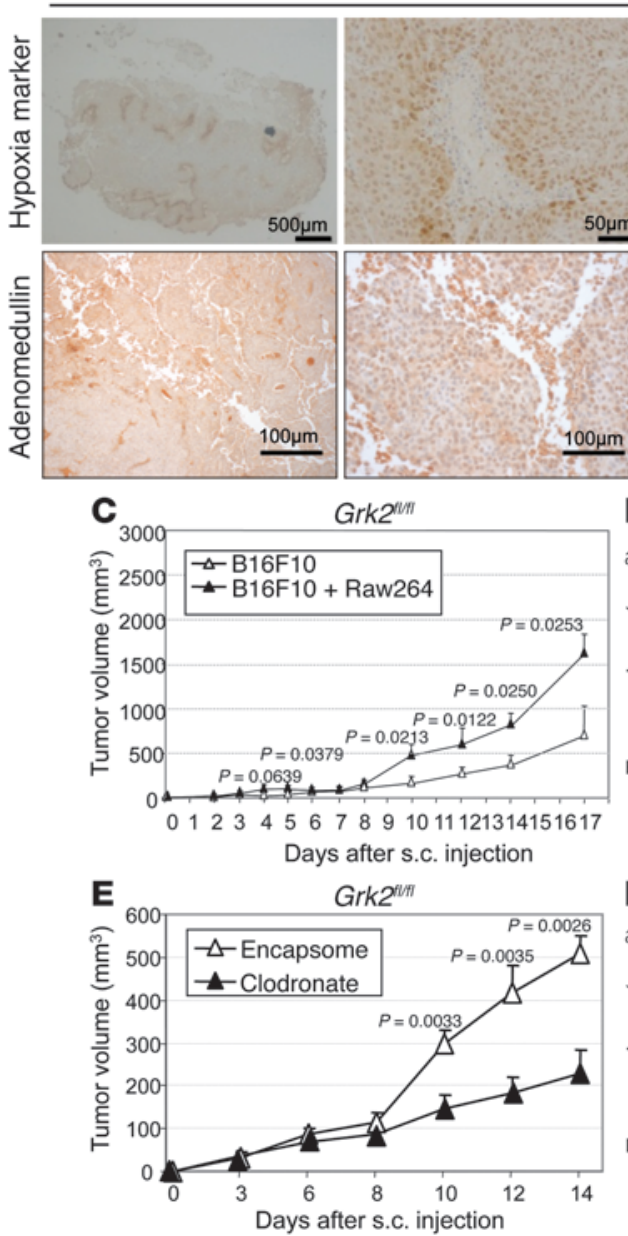

Tie2Cre-Grk2 $2^{\text {min }}$

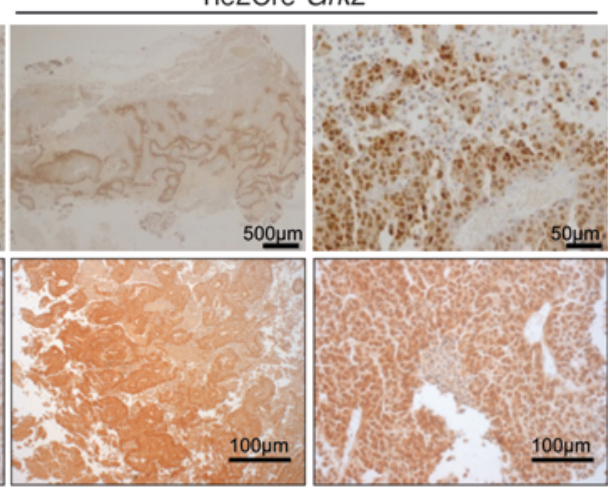

D

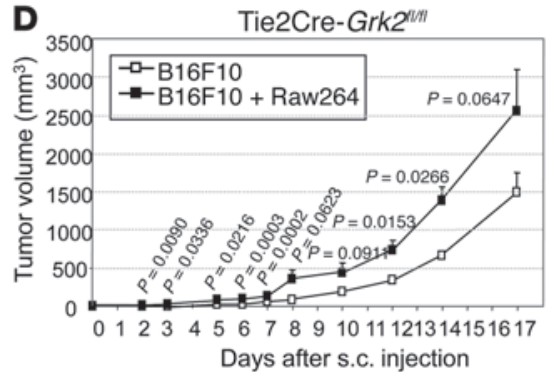

$\mathbf{F}$

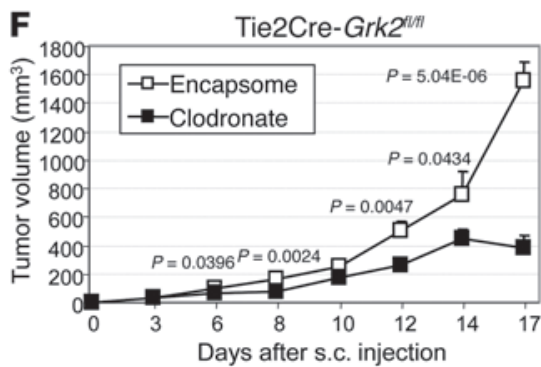

\section{Figure 8}

Absence of endothelial GRK2 expression enhances monocyte migration in vitro and promotes tumor growth in a macrophage-dependent manner. (A) Enhanced RAW267.4 macrophage recruitment in response to conditioned media from ECs lacking GRK2. 48-hour-conditioned media of MLECs from WT Grk2+/+ or Tie2Cre-Grk2 $2^{f / f l}$ mice were used as chemoattractant in Transwell migration assays (data from 3 to 4 independent experiments performed in duplicate). (B) Lack of endothelial GRK2 contributes to enhance intratumoral hypoxia and adrenomedullin expression. B16F10 melanoma cells were subcutaneously injected in mice, and tumors were removed 17 days after and stained with the anti-pimonidazole-based adducts FITC-Mab1 antibody for detection of hypoxia or anti-adrenomedullin antibody, as detailed in Methods, and hematoxylin counterstained. Scale bar: $500 \mu \mathrm{m}$ (hypoxia, first and third images); $50 \mu \mathrm{m}$ (hypoxia, second and fourth images); $100 \mu \mathrm{m}$ (adrenomedullin). (C and D) M2-polarized macrophages enhance tumor growth. B16F10 melanoma cells were subcutaneously injected in WT or Tie2Cre-Grk2 ${ }^{f / f f l}$ mice in the presence or absence of IL4-pretreated RAW267.4 cells as described in Methods. Sizes of tumors implanted in WT mice equal those in Tie2Cre-Grk2 $2^{f / f l}$ mice in control conditions when tumor cells in the formers are implemented with macrophages. (E and $\mathbf{F}$ ) Effects of clodronate-promoted depletion of macrophages on tumor growth. Tumor-bearing mice were treated with control liposomes (encapsome) or clodronate-encapsulated liposomes by intraperitoneal injection starting 24 hours before tumor cell inoculation, followed by treatments every 4 days. Macrophages are involved in the higher growth of tumor cells implanted in Tie2Cre-Grk2 ${ }^{\text {fl/fl }}$ mice compared to WT animals, as such differential growth is abrogated in clodronate-treated Tie2Cre-Grk2 $2^{f / f l}$ mice. Five to eight animals were analyzed for each condition. between WT and Tie2Cre-Grk $2^{f / f l}$ mice in control conditions (Figure 8, E and F), strongly suggesting that the enhanced recruitment of macrophages occurring upon endothelial GRK2 downmodulation played a relevant role in fueling tumor development.

Tumor-derived factors reduce the expression of GRK2 in the endothelium of tumor microvasculature. We reasoned that tumor cells might harness the role of GRK2 in vessel stability by actively promoting its downregulation in the endothelium. Remarkably, both
B16F10 melanoma and human breast cancer cell lines MCF7 and Hs578T, but not normal mammary 184B5 cells, significantly reduced the amount of GRK2 protein expressed in WT MLECs after 24 hours of coculture (Figure 9A). Such downregulation was even more pronounced and rapid in Grk2+/- MLECs. Analysis of GRK2 levels in gene expression databases for ECs isolated from normal resting, regenerating, and malignant tissues or contexts (32) indicated that normal ECs from regenerating 
A
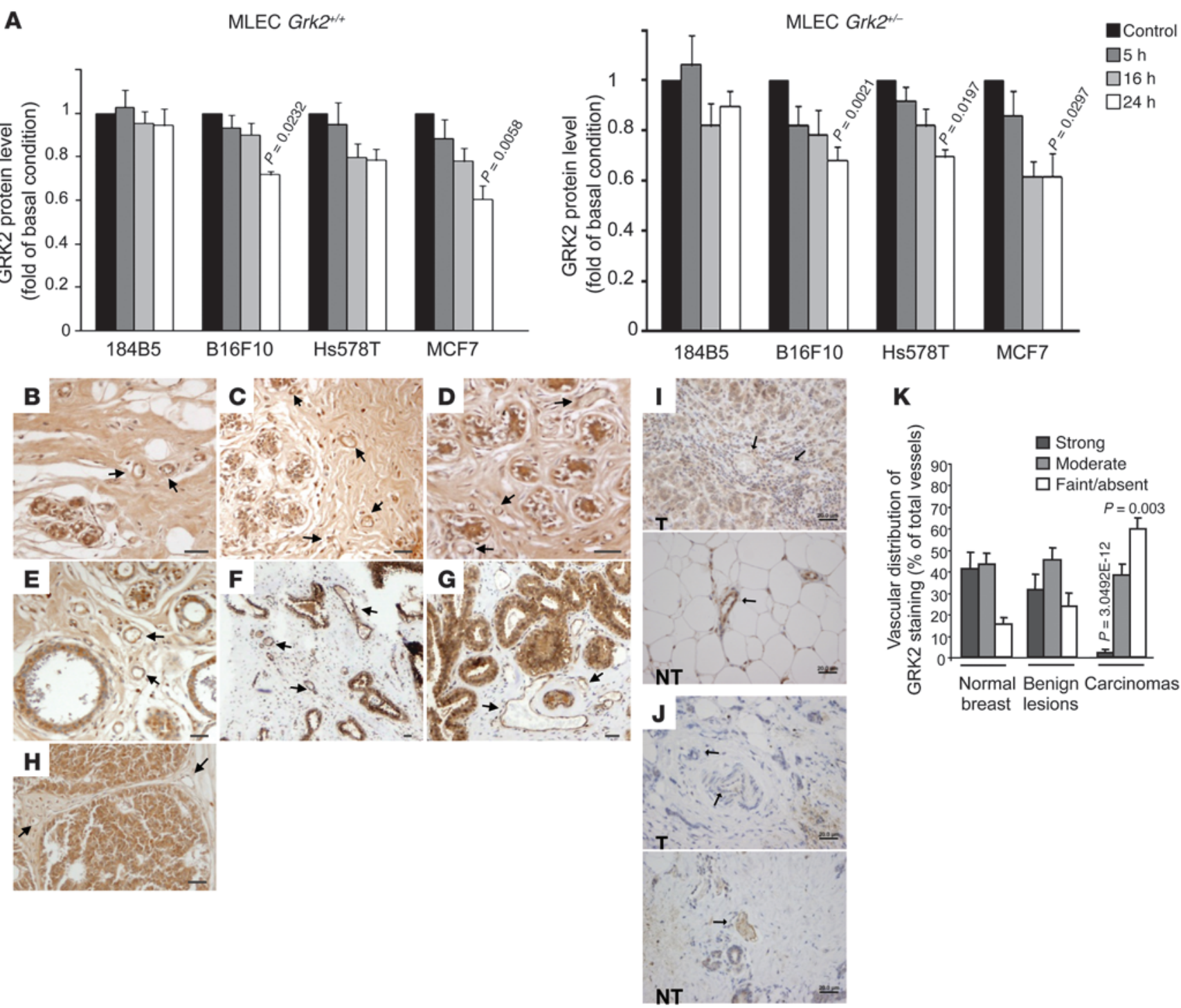

$\mathbf{K}$
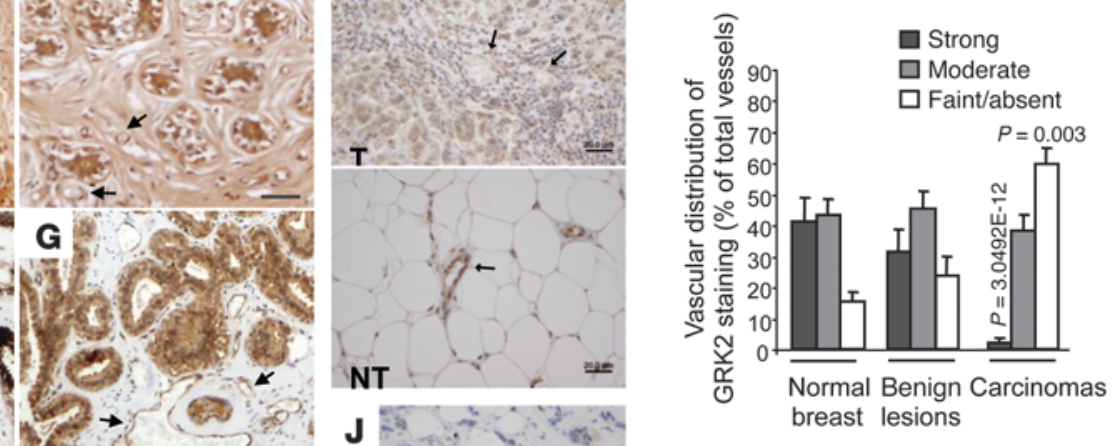

Figure 9

Tumor-derived factors reduce the expression of GRK2 in the endothelium of tumor microvasculature. (A) GRK2 downregulation in MLECs exposed to factors secreted by tumoral but not by nontransformed cell lines. WT or GRK2+/- derived MLECs were cocultured with the different malignant or normal mammary (184B5) cell lines as described in Methods ( $n=3-4$ independent experiments). (B-J) GRK2 expression is markedly decreased in tumor EC cells of breast cancer patients. Immunohistochemical staining of normal and different benign and malignant lesions of the mammary gland reveals that vessels from nontumoral tissues (B-G) show a noticeable signal for GRK2 at the endothelial layer, while tumoral vessels of breast carcinomas $(\mathbf{H}-\mathbf{J})$ display lower or even undetectable GRK2 levels. Normal vessels at the tumor margin retain GRK2 staining. Blood vessels were identified by the presence of blood cells and by anatomic features. Representative vessels are indicated with arrows. (B-D) Normal breast, (E) mild hyperplasia, (F) fibroadenoma, (G) adenosis, and (H-J) breast carcinomas samples were analyzed. T, tumoral area; NT, nontumoral area. (K) Relative vessel immunoreactivity was evaluated by two investigators in a blinded fashion and classified as strong, moderate, or faint/absent GRK2-expressing vessels. The percentage ratio of each vessel group to total vessels analyzed in each breast tissue condition was represented. The number of vessels analyzed was 73 in 7 cases of normal breast tissue, 139 in 14 cases of benign lesions, and 138 in 35 patients of breast carcinoma. $P$ values compare normal cells or normal breast tissues. Scale bar: $20 \mu \mathrm{m}$.

mouse liver upregulate GRK2 expression compared with resting liver ECs (Supplemental Figure 8A), whereas activated human microvascular ECs cocultured with human colorectal cancer LS180 cells in the presence of blood components displayed GRK2 downregulation compared with quiescent ECs (Supplemental Figure 8B) (33). As such an experimental setting mimics the hematogenous microenvironment of tumors caused by a leaky vasculature, we analyzed GRK2 expression in tumoral vessels of different human breast cancer samples (Figure 9, B-K). GRK2 staining was notable in vessels of normal breast tissue and of benign lesions such as adenosis or fibroadenoma. The distribution and the intensity of vascular GRK2 staining were similar in all normal and benign breast tissues examined, showing a high proportion of vessels (>80\%) with a moderate/intense signal for GRK2 (Figure 9, B-K). In marked contrast, approximately $60 \%$ of vessels of different breast carcinoma tumors 
displayed only weak signal or no signal for GRK2, and a focal pattern of GRK2 staining lining positive vessels was frequently observed. Interestingly, lack of GRK2 staining is predominantly associated with intratumoral vessels, while vessels in the normal tissue surrounding the tumor core are clearly immunoreactive for GRK2 (Figure 9, B-K). In addition to the vessel-like pattern of GRK2 staining, GRK2 expression was detected in the normal epithelial layer of mammary ducts and in the tumor cells themselves, albeit at variable levels (Figure 9, B-K).

\section{Discussion}

We demonstrate herein that GRK2 plays a crucial role in physiological and tumoral angiogenesis. First, our results indicate that GRK2 is involved in counterbalancing the action of different angiogenic growth factors responsible for the activation of ECs. Such an activation phase involves the orchestration of migratory and proliferative responses by 2 different types of ECs (tip and stalk cells) found at angiogenic sprouts. Tip cells are highly motile ECs that respond to attractive and repulsive cues, whereas stalk cells proliferate and facilitate tube lumen formation (1). We found that GRK2 downregulation in MLECs causes enhanced signaling to ERK and AKT cascades in response to S1P and VEGF, leading to increased migration. In addition, Grk2 ${ }^{+/-}$MLECs also secrete more PDGF-BB, which is a distinctive hallmark of motile tip cells (26). Noteworthily, the impact of GRK2 levels in EC migration in response to $\mathrm{S} 1 \mathrm{P}$ opposes that described in epithelial cells and fibroblasts (34), thus strengthening the concept that the effects of GRK2 on cell migration would depend on the precise stimuli or cell type considered (35). The influence of environmental cues on the role of GRK2 in migration might be particularly relevant during retinal vascular development. Thus, despite GRK2 downmodulation enhances in vitro migration of ECs toward fibronectin and proliferation is not impaired, radial expansion of retinal vasculature in mutant mice is reduced. Tip cells produce numerous filopodia that help to sense and respond to guidance cues during migration. These cytoskeletal protrusions closely align to the fibronectin-rich astrocytic network, which provides a transient and focally distributed matrix for endothelial tip cell guidance in combination with soluble chemotactic gradients (i.e., VEGF) $(28,36)$. Defective filopodia formation in tip cells lacking GRK2 expression might underpin delayed advance of ECs over a retinal astrocytic template, without interfering with the potential for migration toward gradients of soluble or nonfocal chemotactic factors that can be less affected by the number/ length of cellular filopodia.

In addition, GRK2 downregulation in ECs alters the endothelial barrier function and causes defective tube formation on Matrigel, a process that depends on profuse cellular polarization and morphogenetic rearrangements. Consistently, in vivo angiogenesis in response to FGF2, VEGF, or S1P was markedly impaired in both global and endothelium-specific Grk2 $2^{+/-}$mice, with unstructured vessels and the presence of disperse aggregations of separate pericytes and ECs. The recruitment of mesenchymal cells into new vessels and their interaction with ECs is mediated by tightly controlled gradients of different factors. PDGF-BB facilitates recruitment to vessels by binding to PDGFR- $\beta$ on pericytes (9) and through stimulation of endothelial production of the pericyte chemoattractant CXCL12 (37). Notably, aberrant endothelial secretion of PDGF-BB and of CXCL12 was found in Grk2 ${ }^{+-}$MLECs. Overall, these results indicate that adequate levels of GRK2 are important not only for the normal course of the activation phase of angiogenesis but also for the resolution phase involving vessel coverage by VSMCs/pericytes and vascular stabilization.

GRK2 as a new modulator of TGF- $\beta$ signaling through ALK1 and ALK5. The TGF- $\beta$ signaling axis is central to the proper integration of angiogenic activation and resolution processes $(4,5,24)$. Cellular responses triggered by TGF- $\beta 1$ in ECs are complex, contributing positively or negatively to endothelium activation in a context-dependent manner. Such duality might be explained by the coexistence of two oppositely acting receptors in ECs, ALK1 and ALK5, and their functional cross-modulation $(24,38)$. We found that MLECs from Grk2+/- mice display enhanced Alk5-Smad2/3 and decreased Alk1-Smad5/8 signaling compared with WT cells and that they are more sensitive than WT cells to TGF- $\beta$-mediated antiproliferative and anti-tube formation signals at doses preferentially targeting ALK5 receptors. The fact that Grk2+/MLECs display an activated phenotype in a signaling context of ALK1-Smad5/8 downregulation and fail to assemble mature vessels supports that ALK1 could be also relevant in processes related to angiogenic resolution as differentiation or mural recruitment (39). Our data suggest that GRK2 levels would alter the balance between ALK1/ALK5 pathways in several ways. First, GRK2 would inhibit the ALK5 pathway at the level of Smad2/3, consistent with the previously reported direct, kinase activity-dependent modulation of this factor by GRK2 in hepatic cells (11). In addition, our results indicate that GRK2 positively contributes to the activation of the ALK1 route downstream of the receptor. It is tempting to suggest that GRK2 downmodulation decreased direct inhibition of Smad2/3 and decreased ALK1 signaling-mediated lateral inhibition of the ALK5 pathway (24) would contribute to the observed unbalance in TGF- $\beta$ signaling toward the ALK5 branch.

In sum, we suggest that GRK2 levels can function as a rheostat of ALK5 and ALK1 receptor signaling pathways in angiogenesis and that alterations in GRK2 activity/levels would disturb signaling from TGF- $\beta$ (and therefore its downstream cross-talk with other angiogenic factors), leading to compromised vascular formation.

Reduced GRK2 expression in endothelium leads to an altered pattern of vessel growth and maturation in the postnatal mouse retina and to severe embryonic vascular malformations. Our results clearly demonstrate a key physiological role of GRK2 as a modulator of vessel outgrowth in the postnatal mouse retina and in the developing vasculature. In the widely used mouse retina angiogenesis experimental model, Tie2Cre-Grk $2^{f l / f l}$ animals display clear alterations in the vascular density and sprouting activity at P9 as well as impaired timing of vascular remodeling at P14 that results in altered vessel architecture. Impaired filopodia formation and sprouting of retinal ECs in Tie2Cre-Grk2 $2^{f / f l}$ mice might be related to intrinsic defects in their ability to build up tubular morphogenesis (Figure 2, $\mathrm{D}$ and $\mathrm{E}$, and Supplemental Figure $2 \mathrm{~F}$ ) and to the restrictive effect of the ALK5-Smad2/3 signaling branch on sprouting (40), which would be strengthened by GRK2 downmodulation. Key to vascular remodeling is the selective branch regression, or pruning, and the stabilization of the remaining vessels, being both processes dependent on proper pericyte/EC interactions $(29,28,41)$. Thus, perturbation of endogenous PDFG-BB gradients by intraocular injection of PDGF-BB during vascular remodeling (at P10) results in the formation of aberrant endothelial-pericyte interactions similar to those observed in P14 retinas of Tie2Cre-Grk $2^{f l / f l}$ mice and leads to altered vascular remodeling (29). It is tempting to suggest that increased secretion of mural cell attractants, such as PDGF 
or CXCL12, by GRK2-deficient ECs could disturb local gradients needed to drive an adequate intercellular apposition of pericytes $(42,43)$. More recently, similar to the features of retinal vascularization observed in Tie2Cre-Grk $2^{f l / f l}$ animals, it has been reported that functional downplay of coating pericytes causes increased retinal vessel density and defective vessel regression in the absence of differences in pericyte recruitment/coverage (41). Overall, this evidence indicates that impaired functional interplay between ECs and pericytes during retinal vasculature development might underpin deficient vessel remodeling and maturation.

Such altered functional interaction between endothelial and mural cells is also detected in other physiological contexts. We report that global GRK2 knockout mouse embryos display marked vascular malformations, with blood vessels of increased size and irregular contours. Strikingly, the endothelia of GRK2-deficient mice were almost devoid of mural cell recruitment, which is determinant for vessel maturation. Such defects in pericyte and smooth muscle cell coating appear to be secondary to a general endothelial dysfunction and not a result of defects in pericyte specification, proliferations or mobilization, since Grk2+/- VSMCs display even higher migration rates toward PDGF than WT cells, in agreement with previous data (13), and no angiogenic phenotype has been described in mice with specific GRK2 deletion in VSMCs (15). Moreover, endothelium-specific ablation of GRK2 also results in a leaky vasculature with defective covering of SMCs and pericytes. Even though SMA-positive cells migrate into the surround-

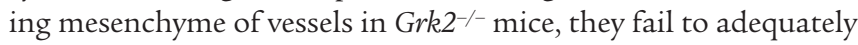
contact ECs. This suggests that the normal gradients of pericyteattracting signals derived from endothelial cells could be altered. Remarkably, similar vascular phenotypes have been reported in the ALK1 (39), endoglin (44), and Smad5 knockout mice (45) or in mice with ablated PDGF-BB/PDGFR $\beta$ axes (9). Vascular abnormalities in $A l k 1^{-/-}$mice may result from the persistence of the activation phase of angiogenesis (39). Our results would strengthen such notion, as GRK2 depletion in ECs promotes hypofunctionality of the ALK1/Smad5 signaling axis in parallel to an immature endothelial phenotype.

Global ablation of GRK2 results in embryonic lethality from E10.5 and is associated with generalized growth retardation and cardiac hypoplasia, although cardiac myocardial-specific knockout animals are viable $(46,47)$. In contrast, endothelial-specific ablation of GRK2 results in viable mice, despite the occurrence of similar vascular alterations. Although we cannot rule out that nonendothelial components in Grk2-/- embryos promote an extrafunctional deterioration of their vasculature to cause lethality in a completely vascular-dependent manner, viability of GRK2 endothelial-knockout mice suggests that lethality of the former requires vascular-independent events. In this regard, it has been reported that GRK2 regulates cell cycle in different ways $(7,48)$ and that its presence is mandatory for cell cycle checkpoints and Smoothened-dependent proliferation and patterning during embryonic development $(48,49)$.

GRK2 downregulation in ECs reduces pericyte coverage of tumoral vessels and potentiates tumor progression. Pathological vascular remodeling is critical for the progression of many diseases, including cancer. Interestingly, we found that accelerated xenograft tumor progression, compared with that in WT animals, occurs upon global systemic hemizygosity and endothelium-restricted GRK2 downregulation, along with immature tumor vessel architecture and increased vessel perimeter and tortuosity. Microvascular density, architec- ture, and maturity are important factors affecting tumor growth $(2,30)$. Poor vascularization has been suggested to lead to excessive hypoxia, which in turn can impel tumor cells to acquire new growth and invasive capabilities (50). Moreover, there is an inverse correlation between the extent of pericyte coverage of tumor vessels and tumor growth progression $(43,51,52)$. Immature vascular plexuses induced by high-PDGF-BB-secreting cancerous cells promote accelerated rates of tumor growth (52). In line with these studies, vessels of tumors growing in Tie2Cre-Grk $2^{f l-}$ mice were also less covered by pericytes compared with those in WT animals, and tumors grown in Tie2Cre-Grk2 $2^{f / f l}$ animals displayed higher infiltration of macrophages and a marked increase of hypoxia and adrenomedullin, known to contribute to enhance tumor progression (53). Thus, our data strongly suggest that the decreased pericyte coverage promoted by GRK2 downregulation in the endothelium would lead to leaky tumor vascularization and consequent hypoxia. This situation, together with the fact that the conditioned media of ECs lacking GRK2 display an enhanced ability to trigger macrophage migration and an altered secretory profile of CXCL12 and other macrophage regulatory factors, would favor attraction, rolling, and diapedesis of macrophages (refs. 54, 55, and Supplemental Figure $7 C)$. Given that tumor-associated macrophages support malignant proliferation, survival, angiogenesis, and stroma remodeling (31), their increased presence would contribute to the higher rate of tumor growth observed in GRK2-deficient mice. Consistent with this notion, depletion of endogenous macrophages in Tie2CreGrk2 $2^{f / f l}$ mice abrogated the enhanced tumor growth observed in these animals compared with that in WT littermates.

Manipulation of tumor-associated angiogenesis represents a promising strategy to limit cancer progression. Different antiangiogenic therapies have been developed, including those targeting VEGF, PDGF, or FGF2 signaling pathways $(1,2)$. Because these pathways also regulate normal vascular remodeling, it is important to identify both new specific signaling cascades in tumor angiogenesis and tumoral vessel markers in order to increase specificity of anticancer treatments. Our data reveal that EC GRK2 is decreased during tumoral, but not normal, angiogenesis, suggesting that GRK2 downmodulation could represent a novel marker for pathological vasculature. In fact, we report that vessels of different breast carcinoma tumors display decreased GRK2 levels. Based on the impact of GRK2 downregulation in vessel maturation, we propose that tumor-driven downregulation of endothelial GRK2 could alter the responsiveness of those vessels sprouting or co-opted within the tumor mass, resulting in an intrinsically altered angiogenic response characterized by excessive endothelial activation, defective transition to maturation, and pericyte detachment. Such tumor-liable endothelia will respond to the extrinsic imbalance of angiogenic factors released by tumors cells, further stimulating a continuously remodeled vasculature that is unable to mature, thereby giving rise to more permeant vessels that ultimately favor tumor growth and dissemination. It has been proposed that pericytes can function as gatekeepers of tumor intravasation (30) and that some antiangiogenic therapies can secondarily disrupt pericyte-endothelial interactions, which results in more aggressive tumors $(2,30)$. Therefore, strategies aiming to reestablish normal vessel architecture could increase perfusion and reduce local invasiveness and cell intravasation (56). In this regard, prevention of GRK2 downregulation in the tumor vasculature would be predicted to facilitate pericyte coverage, switching ECs toward maturation and vessel stabilization. 
Overall, our data suggest that GRK2 downregulation would be a relevant event in the angiogenic switch triggered by tumor cells by favoring a permissive microenvironment for tumor progression. Notably, GRK2 levels are also altered in different malignant mammary cell lines (57), and enhanced GRK2 levels increase epithelial cell motility $(34,58)$, stressing that cell type-specific modulation of this kinase may participate in cancer development. As tumor progression is the result of the interplay between malignant cells themselves and their surrounding microenvironment, our results suggest that opposite changes in GRK2 expression may occur in transformed epithelial cells and in the tumor endothelium to synergistically promote tumor cell invasiveness and intravasation.

\section{Methods}

Animal experiments. Systemic GRK2 knockout mice (which die at early gestational age) and animals hemizygous for this kinase ( $\mathrm{Grk2} 2^{+-}$animals) have been generated previously (46). Endothelium-specific GRK2 knockout mice (Tie2Cre-Grk2 $2^{f / f l}$ mice) were generated by mating GRK2-floxed mice (Grk2 $2^{f / f l}$ mice) (47) and transgenic endothelial Tie2Cre animals, in which expression of Cre recombinase is driven by the endothelial-specific promoter of the Tek (Tie2) receptor. We also generated "hybrid" animal models, in which individual animals express different extraendothelial GRK2 protein levels in combination with distinct endothelial GRK2 content, by crossbreeding systemic hemizygous $\left(G r k 2^{+/-}\right)$mice with endothelial-restricted knockout Tie2Cre-Grk $2^{f / f l}$ mice. These animal models, the Matrigel plug assay for assessing angiogenesis in vivo, and tumor implantation and characterization are described in detail in the Supplemental Methods.

Cell culture and coculture assays. MCF7, Hs578T, RAW267.4, and B16F10 cells (ATCC) were maintained in DMEM supplemented with $10 \%(\mathrm{v} / \mathrm{v})$ fetal bovine serum at $37^{\circ} \mathrm{C}$ in a humidified $5 \% \mathrm{CO}_{2}$ atmosphere. $184 \mathrm{~B} 5$ cells were cultured in DMEM/F12-HAMS medium containing 5\% horse serum, $10 \mu \mathrm{g} / \mathrm{ml}$ insulin, $20 \mathrm{ng} / \mathrm{ml} \mathrm{EGF,} 100 \mathrm{ng} / \mathrm{ml}$ cholera toxin, and $0.5 \mu \mathrm{g} / \mathrm{ml}$ hydrocortisone. Primary MLECs or VSMCs were obtained from mice expressing different dosages of systemic and/or endothelial GRK2, as detailed in Supplemental Methods. Cell migration, proliferation, cytokine secretion, signaling, and in vitro tube formation assays were performed as described in the Supplemental Methods; these are the assays in which different transformed (B16F10, MCF7, and Hs578T) and nontransformed (184B5) cell lines were cocultured with ECs (MLECs).

Immunohistochemistry. Matrigel plugs, implanted tumors, postnatal retinas, embryonic and adult mouse tissues, and human breast cancer samples were processed for histological analysis and immunohistochemistry to analyze vascular structures, as detailed in the Supplemental Methods, using different specific antibodies in order to label mural and ECs populations, GRK2 and adrenomedullin expression, hypoxic status, or macrophage infiltration. Human breast tissue samples were obtained from tissue array collections
(Progression TMA breast carcinoma catalog CC08-00, Biomax, Cybrdi) and from patients recruited at the Hospital Universitario La Paz after approval by the Ethics Committee for Clinical Research of this institution.

Statistics. Data in all figures are expressed as mean \pm SEM. All results were confirmed in at least 2 separate experiments. Data were analyzed using Student's $t$ test. Two-tailed $P<0.05$ was considered statistically significant.

Study approval. The different transgenic mouse models used in this study were housed and bred following all established regulatory standards, and all the experiments were performed in accordance with guidelines of the European Convention for the Protection of Vertebrate Animals used for Experimental and Other Scientific Purposes (Directive 86/609/EEC) and with the authorization of the Bioethical Committee of the Universidad Autónoma de Madrid. Breast cancer samples from patients recruited at the Hospital Universitario La Paz were used for immunochemistry studies after approval by the Ethics Committee for Clinical Research of this institution. At the time of surgical patients were informed and provided consent that the excess of the resected sample could be used for research purposes after diagnosis completion. A specific written consent from patients to participate in this particular study was not necessary since no additional sample was collected.

\section{Acknowledgments}

Our laboratory is funded by grants from Ministerio de Educación y Ciencia (SAF2011-23800), Fundación Ramón Areces, The Cardiovascular Network (RECAVA) of Ministerio Sanidad y Consumo-Instituto Carlos III (RD06-0014/0037 and RD12/0042/0012), and Comunidad de Madrid (S-2010/BMD2332) to F. Mayor Jr. and Instituto Carlos III (PI11/00859), Fundación Ramón Areces, and Fundación Rodríguez Pascual to P. Penela. Marta Mendiola is supported by a postdoctoral research contract from Fondo de Investigación Sanitaria ("Sara Borrell" Programme), Instituto de Salud Carlos III. We thank W.J. Koch for help with the mouse models, Alicia García-Arroyo for helpful advice and insights, V. Labrador for confocal microscopy assistance and image analysis, and P. Ramos, A. Santos, and M. Sanz for technical assistance.

Received for publication October 12, 2012, and accepted in revised form August 15, 2013.

Address correspondence to: Federico Mayor Jr. or Petronila Penela, Centro de Biología Molecular "Severo Ochoa," C/ Nicolás Cabrera 1, Universidad Autónoma de Madrid, 28049 Madrid, Spain. Phone: 34.91.196.4626; Fax: 34.91.196.4420; E-mail: fmayor@cbm.uam.es (F. Mayor Jr.). Phone: 34.91.196.4640; Fax: 34.91.196.4420; E-mail: ppenela@cbm.uam.es (P. Penela).
1. Carmeliet P, Jain RK. Molecular mechanisms and clinical applications of angiogenesis. Nature. 2011; 473(7347):298-307.

2. Weis SM, Cheresh DA. Tumor angiogenesis: molecular pathways and therapeutic targets. Nat Med.2011; 17(11):1359-1370.

3. Kiefer F, Siekmann AF. The role of chemokines and their receptors in angiogenesis. Cell Mol Life Sci. 2011; 68(17):2811-2830.

4. van Meeteren LA, Goumans MJ, ten Dijke P. TGF- $\beta$ receptor signaling pathways in angiogenesis; emerging targets for anti-angiogenesis therapy. Curr Pharm Biotechnol. 2011;12(12):2108-2120.

5. Holderfield MT, Hughes CC. Crosstalk between vascular endothelial growth factor, notch, and transforming growth factor-beta in vascular morphogenesis. Circ Res. 2008;102(6):637-652.
6. Ribas $\mathrm{C}$, et al. The $\mathrm{G}$ protein-coupled receptor kinase (GRK) interactome: role of GRKs in GPCR regulation and signaling. Biochim Biophys Acta. 2007; 1768(4):913-922.

7. Penela P, Murga C, Ribas C, Lafarga V, Mayor F Jr. The complex $G$ protein-coupled receptor kinase 2 (GRK2) interactome unveils new physiopathological targets. Br J Pharmacol. 2010;160(4):821-832.

8. Kovacs JJ, Hara MR, Davenport CL, Kim J, Lefkowitz RJ. Arrestin development: emerging roles for beta-arrestins in developmental signaling pathways. Dev Cell. 2009;17(4):443-458.

9. Hellberg C, Ostman A, Heldin CH. PDGF and vessel maturation. Recent Results Cancer Res. 2010; 180:103-114.

10. Wu JH, Goswami R, Kim LK, Miller WE, Peppel K, Freedman NJ. The platelet-derived growth fac- tor receptor-beta phosphorylates and activates $\mathrm{G}$ protein-coupled receptor kinase-2. A mechanism for feedback inhibition. J Biol Chem. 2005; 280(35):31027-31035.

11. Ho J, Cocolakis E, Dumas VM, Posner BI, Laporte SA, Lebrun JJ. The G protein-coupled receptor kinase-2 is a TGF $\beta$-inducible antagonist of TGF $\beta$ signal transduction. EMBO J. 2005;24(18):3247-3258.

12. D'Souza KM, et al. G protein-coupled receptor kinase- 2 is a novel regulator of collagen synthesis in adult human cardiac fibroblasts. J Biol Chem. 2011; 286(17):15507-15516.

13. Peppel K, et al. Overexpression of G protein-coupled receptor kinase- 2 in smooth muscle cells reduces neointimal hyperplasia. J Mol Cell Cardiol. 2002; 34(10):1399-1409.

14. Morris GE, Nelson CP, Standen NB, Challiss 
RA, Willets JM. Endothelin signalling in arterial smooth muscle is tightly regulated by G proteincoupled receptor kinase 2. Cardiovasc Res. 2010; 85(3):424-433.

15. Cohn HI, et al. Inhibition of vascular smooth muscle $\mathrm{G}$ protein-coupled receptor kinase 2 enhances $\alpha 1 \mathrm{D}$-adrenergic receptor constriction. Am J Physiol Heart Circ Physiol. 2008;295(4):H1695-H1704.

16. Liu S, Premont RT, Kontos CD, Zhu S, Rockey DC. A crucial role for GRK2 in regulation of endothelial cell nitric oxide synthase function in portal hypertension. Nat Med. 2005;11(9):952-958.

17. Nishishita T, Lin PC. Angiopoietin 1, PDGF-B, and TGF- $\beta$ gene regulation in endothelial cell and smooth muscle cell interaction. J Cell Biochem. 2004; 91(3):584-593.

18. Wu X, Ma J, Han JD, Wang N, Chen YG. Distinct regulation of gene expression in human endothelial cells by TGF- $\beta$ and its receptors. Microvasc Res. 2006;71(1):12-19.

19. Shao ES, Lin L, Yao Y, Boström KI. Expression of vascular endothelial growth factor is coordinately regulated by the activin-like kinase receptors 1 and 5 in endothelial cells. Blood. 2009;114(10):2197-2206.

20. Taylor LM, Khachigian LM. Induction of plateletderived growth factor B-chain expression by transforming growth factor- $\beta$ involves transactivation by Smads. J Biol Chem. 2000;275(22):16709-16716.

21. Guo J, et al. TGF $\beta$-induced GRK2 expression attenuates AngII-regulated vascular smooth muscle cell proliferation and migration. Cell Signal. 2009; 21(6):899-905.

22. Pepper MS, Vassalli JD, Orci L, Montesano R. Biphasic effect of transforming growth factorbeta 1 on in vitro angiogenesis. Exp Cell Res. 1993; 204(2):356-363.

23. English D, et al. Sphingosine 1-phosphate released from platelets during clotting accounts for the potent endothelial cell chemotactic activity of blood serum and provides a novel link between hemostasis and angiogenesis. FASEB J. 2000; 14(14):2255-2265.

24. Goumans $M J$, et al. Activin receptor-like kinase (ALK) 1 is an antagonistic mediator of lateral TGF $\beta /$ ALK5 signaling. Mol Cell. 2003;12(4):817-828.

25. Au P, et al. Paradoxical effects of PDGF-BB overexpression in endothelial cells on engineered blood vessels in vivo. Am J Pathol. 2009;175(1):294-302.

26. Hellström M, Kalén M, Lindahl P, Abramsson A, Betsholtz C. Role of PDGF-B and PDGFR- $\beta$ in recruitment of vascular smooth muscle cells and pericytes during embryonic blood vessel formation in the mouse. Development. 1999;126(14):3047-3055.

27. Stahl A, et al. The mouse retina as an angiogenesis model. Invest Ophthalmol Vis Sci. 2010;51(6):2813-2826.

28. Fruttiger M. Development of the retinal vasculature. Angiogenesis. 2007;10(2):77-88.

29. Benjamin LE, Hemo I, Keshet E. A plasticity window for blood vessel remodelling is defined by pericyte coverage of the preformed endothelial network and is regulated by PDGF-B and VEGF. Development. 1998;125(9):1591-1598.

30. Gerhardt H, Semb H. Pericytes: gatekeepers in tumour cell metastasis? J Mol Med (Berl). 2008; 86(2):135-144.

31. Condeelis J, Pollard JW. Macrophages: obligate partners for tumor cell migration, invasion, and metastasis. Cell. 2006;124(2):263-266.

32. Seaman S, Stevens J, Yang MY, Logsdon D, GraffCherry C, St Croix B. Genes that distinguish physiological and pathological angiogenesis. Cancer Cell. 2007;11(6):539-554.

33. Läubli H, Spanaus KS, Borsig L. Selectin-mediated activation of endothelial cells induces expression of CCL5 and promotes metastasis through recruitment of monocytes. Blood. 2009;114(20):4583-4591.

34. Penela $P$, et al. G protein-coupled receptor kinase 2 positively regulates epithelial cell migration. $E M B O J$. 2008;27(8):1206-1218.

35. Penela P, Ribas C, Aymerich I, Mayor F Jr. New roles of $G$ protein-coupled receptor kinase 2 (GRK2) in cell migration. Cell Adh Migr. 2009;3(1):19-23.

36 . Stenzel D, et al. Integrin-dependent and -independent functions of astrocytic fibronectin in retinal angiogenesis. Development. 2011;138(20):4451-4463.

37. Song $N$, et al. Overexpression of platelet-derived growth factor-BB increases tumor pericyte content via stromal-derived factor- $1 \alpha /$ CXCR 4 axis. Cancer Res. 2009;69(15):6057-6064.

38. Carvalho RL, et al. Compensatory signalling induced in the yolk sac vasculature by deletion of TGF $\beta$ receptors in mice. J Cell Sci. 2007;120(pt 24):4269-4277.

39. Oh SP, et al. Activin receptor-like kinase 1 modulates transforming growth factor- $\beta 1$ signaling in the regulation of angiogenesis. Proc Natl Acad Sci U S A. 2000;97(6):2626-2631.

40. Mallet C, Vittet D, Feige JJ, Bailly S. TGF $\beta 1$ induces vasculogenesis and inhibits angiogenic sprouting in an embryonic stem cell differentiation model: respective contribution of ALK1 and ALK5. Stem Cells. 2006;24(11):2420-2427.

41. Simonavicius N, et al. Pericytes promote selective vessel regression to regulate vascular patterning. Blood. 2012;120(7):1516-1527.

42. Lindblom P, et al. Endothelial PDGF-B retention is required for proper investment of pericytes in the microvessel wall. Genes Dev. 2003;17(15):1835-1840. 43. Abramsson A, Lindblom P, Betsholtz C. Endothelial and nonendothelial sources of PDGF-B regulate pericyte recruitment and influence vascular pattern. J Clin Invest. 2003;112(8):1142-1151.

44. Li DY, et al. Defective angiogenesis in mice lacking endoglin. Science. 1999;284(5419):1534-1537.

45. Yang $X$, et al. Angiogenesis defects and mesenchymal apoptosis in mice lacking SMAD5. Development. 1999;126(8):1571-1580.

46. Jaber $M$, et al. Essential role of $\beta$-adrenergic receptor kinase 1 in cardiac development and function. Proc Natl Acad Sci U S A. 1996;93(23):12974-12979.

47. Matkovich SJ, et al. Cardiac-specific ablation of G-protein receptor kinase 2 redefines its roles in heart development and $\beta$-adrenergic signaling. Circ Res. 2006;99(9):996-1003.

48. Jiang X, Yang P, Ma L. Kinase activity-independent regulation of cyclin pathway by GRK2 is essential for zebrafish early development. Proc Natl Acad Sci US A. 2009;106(25):10183-10188.

49. Philipp $M$, et al. Smoothened signaling in vertebrates is facilitated by a $\mathrm{G}$ protein-coupled receptor kinase. Mol Biol Cell. 2008;19(12):5478-5489.

50. Voss MJ, Moller MF, Powe DG, Niggemann B, Zanker KS, Entschladen F. Luminal and basallike breast cancer cells show increased migration induced by hypoxia, mediated by an autocrine mechanism. BMC Cancer. 2011;11:158.

51. Xian X, et al. Pericytes limit tumor cell metastasis. J Clin Invest. 2006;116(3):642-651.

52. Nissen LJ, et al. Angiogenic factors FGF2 and PDGF$\mathrm{BB}$ synergistically promote murine tumor neovascularization and metastasis. J Clin Invest. 2007; 117(10):2766-2777.

53. Chen $\mathrm{P}$, et al. Tumor-associated macrophages promote angiogenesis and melanoma growth via adrenomedullin in a paracrine and autocrine manner. Clin Cancer Res. 2011;17(23):7230-7239.

54. Belperio JA, et al. CXC chemokines in angiogenesis. J Leukoc Biol. 2000;68(1):1-8.

55. Dirkx AE, Oude Egbrink MG, Wagstaff J, Griffioen AW. Monocyte/macrophage infiltration in tumors: modulators of angiogenesis. J Leukoc Biol. 2006; 80(6):1183-1196.

56. Goel S, et al. Normalization of the vasculature for treatment of cancer and other diseases. Physiol Rev. 2011;91(3):1071-1121.

57. Salcedo A, Mayor F Jr, Penela P. Mdm2 is involved in the ubiquitination and degradation of G-protein-coupled receptor kinase 2. EMBO J. 2006; 25(20):4752-4762.

58. Lafarga V, Aymerich I, Tapia O, Mayor F Jr, Penela P. A novel GRK2/HDAC6 interaction modulates cell spreading and motility. EMBO J. 2012; 31(4):856-869. 